\title{
INTEGRAL FOLIATED SIMPLICIAL VOLUME OF HYPERBOLIC 3-MANIFOLDS
}

\author{
CLARA LÖH AND CRISTINA PAGLIANTINI
}

\begin{abstract}
Integral foliated simplicial volume is a version of simplicial volume combining the rigidity of integral coefficients with the flexibility of measure spaces. In this article, using the language of measure equivalence of groups we prove a proportionality principle for integral foliated simplicial volume for aspherical manifolds and give refined upper bounds of integral foliated simplicial volume in terms of stable integral simplicial volume. This allows us to compute the integral foliated simplicial volume of hyperbolic 3-manifolds. This is complemented by the calculation of the integral foliated simplicial volume of Seifert 3-manifolds.
\end{abstract}

\section{INTRODUCTION}

Integral foliated simplicial volume is a version of simplicial volume combining the rigidity of integral coefficients with the flexibility of measure spaces. If $M$ is an oriented closed connected manifold, then the integral foliated simplicial volume $|M|$ fits into the sandwich

$$
\|M\| \leq|M| \leq\|M\|_{\mathbb{Z}}^{\infty},
$$

where $\|M\|$ is the classical simplicial volume [13] and where $\|M\|_{\mathbb{Z}}^{\infty}$ is the stable integral simplicial volume (stabilised over all finite coverings of $M$ ).

Gromov [15, p. 305f] suggested a definition of integral foliated simplicial volume and an upper estimate of $L^{2}$-Betti numbers in terms of integral foliated simplicial volume, which was confirmed by Schmidt [28]. It is an open problem whether integral foliated simplicial volume coincides with simplicial volume in the case of aspherical manifolds; an affirmative answer would show that aspherical manifolds with vanishing simplicial volume have vanishing Euler characteristic, which is a long-standing open problem [14, p. 232]. The only cases in which the integral foliated simplicial volume has been computed are manifolds that split off an $S^{1}$-factor and simply connected manifolds [28, Chapter 5.2]. Moreover, Sauer proved an upper bound of a related invariant in terms of minimal volume [27, Section 3].

In the present article, we will prove the following:

Theorem 1.1 (integral foliated simplicial volume of hyperbolic 3-manifolds). For all oriented closed connected hyperbolic 3-manifolds $M$ the integral foliated simplicial volume and the simplicial volume of $M$ coincide:

$$
|M|=\|M\|=\frac{\operatorname{vol}(M)}{v_{3}} .
$$

Here, $v_{3}$ denotes the maximal volume of ideal geodesic 3 -simplices in $\mathbb{H}^{3}$.

Date: June 29, 2018. (C) C. Löh, C. Pagliantini 2013, MSC 2010 classification: 57M27, 57M50, 20F67, 55N99. 
The second equality in Theorem 1.1 is the classic proportionality principle for hyperbolic manifolds of Gromov [13] and Thurston [31], which also holds in more generality $7,10,11,5,29,4,9,1,19]$.

The proof of Theorem 1.1 consists of the following steps: Using the language of measure equivalence of groups and techniques of Bader, Furman and Sauer [1] we show that, similarly to classical simplicial volume, also integral foliated simplicial volume of aspherical manifolds satisfies a proportionality principle with respect to certain parameter spaces (see Section 5 and Section 4 for the definitions):

Theorem 1.2 (proportionality principle for integral foliated simplicial volume). Let $M$ and $N$ be oriented closed connected aspherical manifolds of the same dimension satisfying $\|M\|>0$ and $\|N\|>0$. Suppose that there exists an ergodic bounded measure equivalence coupling $(\Omega, \mu)$ of the fundamental groups $\Gamma$ and $\Lambda$ of $M$ and $N$, respectively; let $c_{\Omega}$ be the coupling index of this coupling.

(1) Then

$$
|M|^{\Lambda \backslash \Omega}=c_{\Omega} \cdot|N|^{\Gamma \backslash \Omega} .
$$

(2) If the coupling $(\Omega, \mu)$ is mixing, then

$$
|M|=c_{\Omega} \cdot|N| .
$$

Considering the coupling of uniform hyperbolic lattices given by the isometry group, we obtain:

Corollary 1.3 (a proportionality principle for integral foliated simplicial volume of hyperbolic manifolds). Let $n \in \mathbb{N}$, and let $\Gamma, \Lambda<G:=\operatorname{Isom}^{+}\left(\mathbb{H}^{n}\right)$ be uniform lattices. Then

$$
\frac{\left|\mathbb{H}^{n} / \Gamma\right|^{G / \Lambda}}{\operatorname{covol}(\Gamma)}=\frac{\left|\mathbb{H}^{n} / \Lambda\right|^{G / \Gamma}}{\operatorname{covol}(\Lambda)}
$$

and

$$
\frac{\left|\mathbb{H}^{n} / \Gamma\right|}{\operatorname{covol}(\Gamma)}=\frac{\left|\mathbb{H}^{n} / \Lambda\right|}{\operatorname{covol}(\Lambda)} .
$$

Therefore, when estimating integral foliated simplicial volume of a hyperbolic manifold, we can call other hyperbolic manifolds for help.

In particular, we obtain the following refinement of the upper bound of integral foliated simplicial volume in terms of stable integral simplicial volume:

Corollary 1.4 (comparing integral foliated simplicial volume and stable integral simplicial volume, hyperbolic case). Let $n \in \mathbb{N}$, and let $M$ and $N$ be oriented closed connected hyperbolic $n$-manifolds. Then

$$
|M| \leq \frac{\operatorname{vol}(M)}{\operatorname{vol}(N)} \cdot\|N\|_{\mathbb{Z}}^{\infty} .
$$

Moreover, we exhibit concrete examples of parameter spaces that realize stable integral simplicial volume as integral foliated simplicial volume: 
Theorem 1.5 (comparing integral foliated simplicial volume and stable integral simplicial volume, generic case). Let $M$ be an oriented closed connected manifold with fundamental group $\Gamma$ and let $S$ be the set of finite index subgroups of $\Gamma$. Then

$$
\|M\|_{\mathbb{Z}}^{\infty}=|M|^{\prod_{\Lambda \in S} \Gamma / \Lambda} .
$$

While Theorem 1.5 is not necessary to prove Theorem 1.1, it is of independent interest in the context of Question 1.7.

As last step in the proof of Theorem 1.1, in dimension 3, we will use the following sequence of hyperbolic manifolds, based on a variation of a result by Francaviglia, Frigerio and Martelli [8]:

Theorem 1.6 (hyperbolic 3-manifolds with small stable integral simplicial volume). There exists a sequence $\left(M_{n}\right)_{n \in \mathbb{N}}$ of oriented closed connected hyperbolic 3-manifolds with

$$
\lim _{n \rightarrow \infty} \frac{\left\|M_{n}\right\|_{\mathbb{Z}}^{\infty}}{\left\|M_{n}\right\|}=1 .
$$

Notice that it is still unknown whether stable integral simplicial volume and simplicial volume coincide for hyperbolic 3-manifolds. Francaviglia, Frigerio, and Martelli [8] proved that in all dimensions bigger than 3 a sequence of hyperbolic manifolds as in Theorem 1.6 does not exist. Therefore, our approach does not allow to compute the integral foliated simplicial volume of higher-dimensional hyperbolic manifolds. Moreover, we do not know whether integral foliated simplicial volume can be different from stable integral simplicial volume for aspherical manifolds with enough finite coverings:

Question 1.7. What is the difference between integral foliated simplicial volume and stable integral simplicial volume of aspherical oriented closed connected manifolds with residually finite fundamental group?

Organisation of this article. In Section 2, we recall the definition and basic properties of (stable integral) simplicial volume. In Section 3 , we construct hyperbolic 3-manifolds with small stable integral simplicial volume, which proves Theorem 1.6. Section 4 is an introduction into integral foliated simplicial volume and basic operations on parameter spaces. We prove the proportionality principles Theorem 1.2 and Corollary 1.3 in Section 5 . Section 6 is devoted to the refinements of the comparison between integral foliated simplicial volume and stable integral simplicial volume and includes a proof of Theorem 1.5. Finally, in Section 7, we complete the proof of Corollary 1.4 and Theorem 1.1. Section 8 contains the computation of integral foliated simplicial volume of Seifert manifolds.

Acknowledgements. We would like to thank Roman Sauer, Roberto Frigerio, Bruno Martelli, and Marco Schmidt for numerous helpful discussions. In particular, we would like to thank Roman Sauer for pointing out a mistake in the first version. This work was partially supported by the Graduiertenkolleg Curvature, Cycles, and Cohomology (Universität Regensburg). The second author was also partially supported by the Swiss National Science Foundation, under the grant 2000200-144373. 


\section{Simplicial Volume And (Stable) integral Simplicial Volume}

In this section we will recall the definition of simplicial volume introduced by Gromov [13, 18] and its integral version, which uses integral homology instead of real homology.

Let $X$ be a topological space. Let $R$ be a normed ring. In this section, we restrict only to the cases $R=\mathbb{R}$ or $\mathbb{Z}$. For $i \in \mathbb{N}$ we denote by $S_{i}(X)$ the set of singular $i$-simplices in $X$, by $C_{i}(X, R)$ the module of singular $i$-chains with $R$-coefficients. The homology of the complex $\left(C_{*}(X, R), \partial_{*}\right)$, where $\partial_{*}$ is the usual differential, is the singular homology $H_{*}(X, R)$ of $X$ with coefficients in $R$.

We endow the $R$-module $C_{i}(X, R)$ with the $\ell^{1}$-norm defined by

$$
\left\|\sum_{\sigma \in S_{i}(X)} a_{\sigma} \cdot \sigma\right\|_{1}^{R}=\sum_{\sigma \in S_{i}(X)}\left|a_{\sigma}\right|,
$$

where $|\cdot|$ is the norm on $R$. We denote the norm $\|\cdot\|_{1}^{\mathbb{R}}$ simply by $\|\cdot\|_{1}$. The norm $\|\cdot\|_{1}^{R}$ descends to a semi-norm on $H_{*}(X, R)$, which is also denoted by $\|\cdot\|_{1}^{R}$ and is defined as follows: if $\alpha \in H_{i}(X, R)$, then

$$
\|\alpha\|_{1}^{R}=\inf \left\{\|c\|_{1}^{R} \mid c \in C_{i}(X, R), \partial c=0,[c]=\alpha\right\} .
$$

Note that $\|\cdot\|_{1}^{\mathbb{Z}}$ on $H_{*}(\cdot, \mathbb{Z})$ is technically not a semi-norm as it is not multiplicative in general (see below).

If $M$ is a closed connected oriented $n$-manifold, then we denote the fundamental class of $M$ by $[M]_{\mathbb{Z}}$, i.e., the positive generator of $H_{n}(M, \mathbb{Z}) \cong \mathbb{Z}$. The change of coefficients homomorphism $H_{n}(M, \mathbb{Z}) \longrightarrow H_{n}(M, \mathbb{R})$ sends the fundamental class to the real fundamental class $[M]_{\mathbb{R}} \in H_{n}(M, \mathbb{R})$ of $M$. The following definition is due to Gromov [13]:

Definition 2.1 ((integral) simplicial volume). The simplicial volume of $M$ is

$$
\|M\|:=\left\|[M]_{\mathbb{R}}\right\|_{1} \in \mathbb{R}_{\geq 0} .
$$

The integral simplicial volume of $M$ is defined as $\|M\|_{\mathbb{Z}}:=\left\|[M]_{\mathbb{Z}}\right\|_{1}^{\mathbb{Z}} \in \mathbb{N}$.

Of course we have the inequality $\|M\| \leq\|M\|_{\mathbb{Z}}$ but in general no equality (for instance, $\left\|S^{1}\right\|=0$ but $\left\|S^{1}\right\|_{\mathbb{Z}}=1$ ). The integral simplicial volume does not behave as nicely as the simplicial volume. For example, it follows from the definition that $\|M\|_{\mathbb{Z}} \geq 1$ for every manifold $M$. Therefore, the integral simplicial volume cannot be multiplicative with respect to finite coverings (otherwise it would vanish on manifolds that admit finite non-trivial selfcoverings, such as $S^{1}$ ). Moreover, as we mentioned before, the $\ell^{1}$-seminorm on integral homology is not really a semi-norm, since the equality $\|n \cdot \alpha\|_{1}^{\mathbb{Z}}=|n| \cdot\|\alpha\|_{1}^{\mathbb{Z}}$, may not hold for all $\alpha \in H_{*}(X ; \mathbb{Z})$ and all $n \in \mathbb{Z}$. Indeed, it is easy to see that $\left\|n \cdot\left[S^{1}\right]_{\mathbb{Z}}\right\|_{1}^{\mathbb{Z}}=1$ for every $n \in \mathbb{Z} \backslash\{0\}$.

We may consider a stable version of the integral simplicial volume:

Definition 2.2 (stable integral simplicial volume). The stable integral simplicial volume of an oriented closed connected manifold $M$ is

$\|M\|_{\mathbb{Z}}^{\infty}:=\inf \left\{\frac{1}{d} \cdot\|\bar{M}\|_{\mathbb{Z}} \mid d \in \mathbb{N}\right.$, there is a $d$-sheeted covering $\left.\bar{M} \rightarrow M\right\}$. 
Since the simplicial volume is multiplicative under finite coverings [18, Proposition 4.1], it is clear that $\|M\| \leq\|M\|_{\mathbb{Z}}^{\infty}$, but in general they are not equal:

Theorem 2.3 ([8, Theorem 2.1]). For every $n \in \mathbb{N}_{\geq 4}$ there exists a constant $C_{n}<1$ such that the following holds: Let $M$ be an oriented closed connected hyperbolic n-manifold. Then

$$
\|M\| \leq C_{n} \cdot\|M\|_{\mathbb{Z}}^{\infty} .
$$

For hyperbolic 3-manifolds it is still an open question whether the simplicial volume and the stable integral simplicial volume are the same: our Theorem 1.6 gives a partial answer.

\section{HyPERBOLIC 3-MANIFOLDS \\ WITH SMALL STABLE INTEGRAL SIMPLICIAL VOLUME}

In this section we will prove Theorem 1.6 following an argument of Francaviglia, Frigerio, and Martelli [8, Corollary 5.16]. For the sake of completeness, we recall some background on triangulations and special complexity.

Definition 3.1 (triangulation). A triangulation of a closed 3-manifold $M$ is a realization of the manifold $M$ as the gluing of finitely many tetrahedra via some simplicial pairing of their faces. Turning to the case of a compact manifold $M$ with non-empty boundary $\partial M$, one can adapt the notion of triangulation: an ideal triangulation of $M$ is a decomposition of its interior $\operatorname{Int}(M)$ into tetrahedra with their vertices removed. An (ideal) triangulation is semi-simplicial if all the edges have distinct vertices.

Definition 3.2 (special complexity). Let $M$ be a compact 3-manifold, possibly with boundary. The special complexity $c_{S}(M)$ of $M$ is the minimal number of vertices in a special spine for $M$.

We refer to the works of Matveev [23, 24] for the definition of special spines and their properties. For our purpose we just need to recall that a special spine is dual to a triangulation. In particular, there is a bijection between the simplices in a triangulation and the vertices in the dual special spine [24, Theorem 1.1.26 and Corollary 1.1.27]. With an abuse of notation we call the true vertices of a special spine in Matveev's definition simply vertices of a special spine.

Theorem 3.3 (Matveev [24, Corollary 1.1.28]). Let $M$ be a compact 3manifold whose interior Int $(M)$ admits a complete finite volume hyperbolic structure. Then there is a bijection between special spines and ideal triangulations of $M$ such that the number of vertices in the special spine is equal to the number of tetrahedra in the corresponding triangulation.

Remark 3.4. Matveev [23] introduced the more general notion of complexity, which involves spines that are not necessarily dual to triangulations and showed that complexity and special complexity are equal for any closed orientable irreducible 3 -manifold distinct from $S^{3}, \mathbb{R P}^{3}$, and $L(3,1)$.

Remark 3.5. Matveev [23] and Martelli [20] used two slighly different definitions of spines but the two notions are equivalent and lead to the same complexity [20, Section 7]. Coherently with [8] we use Martelli's definition. 
Remark 3.6. Let $M$ be an oriented closed connected manifold and $T$ be a semi-simplicial triangulation of $M$. Fixing an order of the vertices and a suitable choice of orientation-preserving parametrisations $\sigma_{1}, \ldots, \sigma_{k}$ of simplices of $T$, then $\sigma_{1}+\cdots+\sigma_{k}$ represents the integral fundamental class of $M$. Therefore the number of tetrahedra in a semi-simplicial triangulation provides an upper bound for the integral simplicial volume of $M$.

In order to adapt the proof of Francaviglia, Frigerio and Martelli [8, Corollary 5.16] we need the following results:

Proposition 3.7. Let $\overline{M_{(5)}}$ be the compactification of the 5-chain link complement $M_{(5)}$. Then we have:

$$
c_{S}\left(\overline{M_{(5)}}\right)=10=\left\|\overline{M_{(5)}}\right\| .
$$

Moreover, the value of $c_{S}\left(\overline{M_{(5)}}\right)$ is realized by a special spine dual to a semisimplicial triangulation.

Proof. The 5-chain link complement has a hyperbolic structure 26] and admits an ideal triangulation with 10 ideal and regular tetrahedra such that each edge has vertices in different cusps [25, Section 5.2]. By the proportionality between simplicial volume and Riemannian volume (which holds both in the compact case [13, 31] and in the cusped case [7, 10, 11, 5]) we have

Moreover, Theorem 3.3 implies

$$
\left\|\overline{M_{(5)}}\right\|=\frac{\operatorname{vol}\left(M_{(5)}\right)}{v_{3}}=10 .
$$

$$
c_{S}\left(\overline{M_{(5)}}\right) \leq 10 \text {. }
$$

The equality follows from the fact that for every oriented connected finite volume hyperbolic 3-manifold $M$ with compactification $\bar{M}$ the inequality $\|\bar{M}\| \leq c_{S}(\bar{M})$ holds. Indeed, an argument by Francaviglia [7, Theorem 1.2 and Proposition 3.8] guarantees that the volume of $M$ can be computed by straightening any ideal triangulation of $M$ and then summing the volume of the straight version of the tetrahedra.

Proposition 3.8. Let $N$ be the compactification of a finite volume oriented connected hyperbolic 3-manifold and suppose that $N$ admits a semi-simplicial triangulation that realizes the value of $c_{S}(N)$. Let $M$ be a manifold obtained by Dehn filling on $N$. Then

$$
\|M\|_{\mathbb{Z}}^{\infty} \leq c_{S}(N) .
$$

Proof. As pointed out in Remark 3.6 we estimate the integral simplicial volume by the number of vertices of the special spine dual to a semi-simplicial triangulation.

From the special spine $P$ dual to a semi-simplicial triangulation of $N$ that realizes $c_{S}(N)$, we construct a special spine for $M$, and hence for its finite coverings, such that the associated triangulations are still semi-simplicial. We obtain $\|M\|_{\mathbb{Z}}^{\infty} \leq c_{S}(N)$ following step by step the argument of Francaviglia, Frigerio, and Martelli [8, Proposition 5.15].

More precisely: Let $T_{1}, \ldots, T_{k}$ be the boundary tori of $N$. For every $i \in\{1, \ldots, k\}$ let $V_{i}$ be an open solid torus in $M \backslash P$ created by Dehn filling on 
the boundary component $T_{i}$. Let $D_{i}^{1}$ and $D_{i}^{2}$ be a pair of parallel meridian discs of $V_{i}$. If $D_{i}^{1}$ and $D_{i}^{2}$ are generic with respect to the cellularization induced by $P$ on $T_{i}$ [8, Lemma 5.9], the spine $P \cup D_{i}^{1} \cup D_{i}^{2}$ is special, dual to a semi-simplicial triangulation, and with $v_{\partial}^{T_{i}}$ vertices added to the ones of $P$. Gluing a pair of parallel discs for each boundary torus $T_{i}$ we obtain a special spine $Q=P \cup \bigcup_{i=1}^{k}\left(D_{i}^{1} \cup D_{i}^{2}\right)$ for $M$ with $c_{S}(N)+\sum_{i=1}^{k} v_{\partial}^{T_{i}}+v_{I}$ vertices, where $v_{I}$ is the number of vertices created by intersections between discs added in different boundary components.

Since $\pi_{1}(M)$ is residually finite, for every $n>0$ there exist $n_{0}>n$, $h>0$ and a regular covering $p: \bar{M} \rightarrow M$ of degree $h n_{0}$ such that, for every $i \in\{1, \ldots, k\}$, the preimage $p^{-1}\left(V_{i}\right)$ consists of $h$ open solid tori $\bar{V}_{i}^{1}, \ldots, \bar{V}_{i}^{h}$ each winding $n_{0}$ times along $V_{i}$ via $p$. The special spine $Q$ of $M$ lifts to a special spine $\bar{Q}:=p^{-1}(Q)$ of $\bar{M}$. In particular, each pair of parallel discs added to $P$ lifts to $n_{0}$ copies of pairs of parallel discs in each open solid torus $\bar{V}_{i}^{j}$. Removing $2 n_{0}-2$ discs for each open solid torus in $\bar{Q}$, we obtain again a special spine $\bar{Q}^{\prime}$ dual to a semi-simplicial triangulation of $\bar{M}$.

By Remark 3.6 we now estimate $\|\bar{M}\|_{\mathbb{Z}}$ with the number of vertices of $\bar{Q}^{\prime}$ :

$$
\|M\|_{\mathbb{Z}}^{\infty} \leq \frac{\|\bar{M}\|_{\mathbb{Z}}}{h n_{0}} \leq c_{S}(N)+\frac{\sum_{i=1}^{k} v_{\partial}^{T_{i}}+v_{I}}{n_{0}} .
$$

Since this holds for every $n>0$ and since $n_{0}>n$, we get the conclusion.

We can now complete the proof of Theorem 1.6.

Proof of Theorem 1.6. Let $\left(M_{n}\right)_{n \in \mathbb{N}}$ be a family of hyperbolic 3-manifolds obtained by Dehn filling on $\overline{M_{(5)}}$. By Thurston's Dehn filling Theorem [31, Chapter 5, page 118] it follows that $\lim _{n \rightarrow \infty} \operatorname{vol}\left(M_{n}\right)=\operatorname{vol}\left(M_{(5)}\right)$, which implies $\lim _{n \rightarrow \infty}\left\|M_{n}\right\|=\left\|\overline{M_{(5)}}\right\|$ using the proportionality principle for hyperbolic manifolds (which holds both in the compact case [13, 31] and in the cusped case $[7,10,11,5])$ Then we have:

$$
1 \leq \frac{\left\|M_{n}\right\|_{\mathbb{Z}}^{\infty}}{\left\|M_{n}\right\|} \leq \frac{c_{S}\left(\overline{M_{(5)}}\right)}{\left\|M_{n}\right\|} \stackrel{n \rightarrow \infty}{\longrightarrow} \frac{c_{S}\left(\overline{M_{(5)}}\right)}{\left\|\overline{M_{(5)}}\right\|}=1 .
$$

where the second inequality follows by Proposition 3.8 , and the last equality by Proposition 3.7 .

\section{INTEGRAL FOLIATED SIMPLICIAL VOLUME}

In the following, we will recall the precise definition of integral foliated simplicial volume by Schmidt [28] and discuss basic facts about the effect of changing parameter spaces.

4.1. Definition of integral foliated simplicial volume. Integral foliated simplicial volume is a version of simplicial volume combining the rigidity of integral coefficients with the flexibility of measure spaces. More precisely, integral foliated simplicial volume is defined via homology with twisted coefficients in function spaces of probability spaces that carry an action of the fundamental group. Background on the convenient category of standard Borel spaces can be found in the book by Kechris [16]. 
Definition 4.1 (parametrised fundamental cycles). Let $M$ be an oriented closed connected $n$-manifold with fundamental group $\Gamma$ and universal covering $\widetilde{M} \longrightarrow M$.

- A standard Borel space is a measurable space that is isomorphic to a Polish space with its Borel $\sigma$-algebra. A standard Borel probability space is a standard Borel space together with a probability measure.

- A standard $\Gamma$-space is a standard Borel probability space $(X, \mu)$ together with a measurable $\mu$-preserving (left) $\Gamma$-action. If the probability measure is clear from the context, we will abbreviate $(X, \mu)$ by $X$.

- If $(X, \mu)$ is a standard $\Gamma$-space, then we equip $L^{\infty}(X, \mu, \mathbb{Z})$ with the right $\Gamma$-action

$$
\begin{aligned}
L^{\infty}(X, \mu, \mathbb{Z}) \times \Gamma & \longrightarrow L^{\infty}(X, \mu, \mathbb{Z}) \\
(f, g) & \longmapsto(x \mapsto(f \cdot g)(x):=f(g \cdot x)) .
\end{aligned}
$$

and we write $i_{M}^{X}$ for the change of coefficients homomorphism

$$
\begin{aligned}
i_{M}^{X}: C_{*}(M, \mathbb{Z}) \cong \mathbb{Z} \otimes_{\mathbb{Z} \Gamma} C_{*}(\widetilde{M}, \mathbb{Z}) \longrightarrow L^{\infty}(X, \mathbb{Z}) \otimes_{\mathbb{Z} \Gamma} C_{*}(\widetilde{M}, \mathbb{Z}) \\
1 \otimes c \longmapsto 1 \otimes c
\end{aligned}
$$

induced by the inclusion $\mathbb{Z} \hookrightarrow L^{\infty}(X, \mathbb{Z})$ as constant functions.

- If $(X, \mu)$ is a standard $\Gamma$-space, then

$$
\begin{aligned}
{[M]^{X}:=H_{n}\left(i_{M}^{X}\right)\left([M]_{\mathbb{Z}}\right) } & \in H_{n}\left(M, L^{\infty}(X, \mathbb{Z})\right) \\
& =H_{n}\left(L^{\infty}(X, \mathbb{Z}) \otimes_{\mathbb{Z} \Gamma} C_{*}(\widetilde{M}, \mathbb{Z})\right)
\end{aligned}
$$

is the $X$-parametrised fundamental class of $M$. All cycles in the chain complex $C_{*}\left(M, L^{\infty}(X, \mathbb{Z})\right)=L^{\infty}(X, \mathbb{Z}) \otimes_{\mathbb{Z} \Gamma} C_{*}(\widetilde{M}, \mathbb{Z})$ representing $[M]^{X}$ are called $X$-parametrised fundamental cycles of $M$.

The integral foliated simplicial volume is now defined as the infimum of $\ell^{1}$-norms over all parametrised fundamental cycles:

Definition 4.2 (integral foliated simplicial volume). Let $M$ be an oriented closed connected $n$-manifold with fundamental group $\Gamma$, and let $(X, \mu)$ be a standard $\Gamma$-space.

- Let $\sum_{j=1}^{k} f_{j} \otimes \sigma_{j} \in C_{*}\left(M, L^{\infty}(X, \mathbb{Z})\right)$ be a chain in reduced form, i.e., the singular simplices $\sigma_{1}, \ldots, \sigma_{k}$ on $\widetilde{M}$ satisfy $\pi \circ \sigma_{j} \neq \pi \circ \sigma_{\ell}$ for all $j, \ell \in\{1, \ldots, k\}$ with $j \neq \ell$ (where $\pi: \widetilde{M} \longrightarrow M$ is the universal covering map). Then we define

$$
\left|\sum_{j=1}^{k} f_{j} \otimes \sigma_{j}\right|^{X}:=\sum_{j=1}^{k} \int_{X}\left|f_{j}\right| d \mu \in \mathbb{R}_{\geq 0} .
$$

- The $X$-parametrised simplicial volume of $M$, denoted by $|M|^{X}$, is the infimum of the $\ell^{1}$-norms of all $X$-parametrised fundamental cycles of $M$.

- The integral foliated simplicial volume of $M$, denoted by $|M|$, is the infimum of all $|M|^{X}$ over all isomorphism classes of standard $\Gamma$-spaces $X$. 
Remark 4.3. Let $\Gamma$ be a countable group. The class of isomorphism classes of standard $\Gamma$-spaces indeed forms a set [28, Remark 5.26].

Remark 4.4. Schmidt's original definition [28, Definition 5.25] requires the actions of the fundamental group on the parameter spaces to be essentially free. However, allowing also parameter spaces with actions that are not essentially free does not change the infimum (Corollary 4.14).

Example 4.5 (trivial parameter space). Let $M$ be an oriented closed connected manifold with fundamental group $\Gamma$. If $(X, \mu)$ is a standard $\Gamma$-space consisting of a single point, then $L^{\infty}(X, \mathbb{Z}) \cong \mathbb{Z}$ (as $\mathbb{Z} \Gamma$-modules with trivial $\Gamma$-action) and so

$$
|M|^{X}=\|M\|_{\mathbb{Z}}
$$

More generally, in combination with Proposition 4.13 (2), we obtain: If $(X, \mu)$ is a standard $\Gamma$-space with trivial $\Gamma$-action, then

$$
|M|^{X}=\|M\|_{\mathbb{Z}}
$$

In particular, if $M$ is simply connected, then $|M|^{X}=\|M\|_{\mathbb{Z}}$ for all standard Borel probability spaces $(X, \mu)$ [28, Proposition 5.29].

Proposition 4.6 (comparison with (integral) simplicial volume 28, Remark 5.23]). Let $M$ be an oriented closed connected $n$-manifold with fundamental group $\Gamma$, and let $(X, \mu)$ be a standard $\Gamma$-space. Then

$$
\|M\| \leq|M|^{X} \leq\|M\|_{\mathbb{Z}} .
$$

Proof. The linear map $L^{\infty}(X, \mathbb{Z}) \longrightarrow \mathbb{R}$ given by integration with respect to $\mu$ maps the constant function 1 to 1 and is norm-non-increasing with respect to the $\ell^{1}$-norm on $L^{\infty}(X, \mathbb{Z})$. From the first property, we easily deduce that the induced map $C_{n}\left(M, L^{\infty}(X, \mathbb{Z})\right) \longrightarrow C_{n}(M, \mathbb{R})$ maps $X$-parametrised fundamental cycles to $\mathbb{R}$-fundamental cycles, and so

$$
\|M\| \leq|M|^{X} .
$$

The inclusion $\mathbb{Z} \hookrightarrow L^{\infty}(X, \mathbb{Z})$ as constant functions is isometric with respect to the $\ell^{1}$-norm and the induced $\operatorname{map} C_{n}(M, \mathbb{Z}) \longrightarrow C_{n}\left(M, L^{\infty}(X, \mathbb{Z})\right)$ maps fundamental cycles to $X$-parametrised fundamental cycles. Hence,

$$
|M|^{X} \leq\|M\|_{\mathbb{Z}}
$$

Remark 4.7 (real coefficients). Arguments analogous to the ones in the proof of the previous proposition show that

$$
|M|^{L^{\infty}(X, \mu, \mathbb{R})}=\|M\|
$$

holds for all oriented closed connected manifolds $M$ and all standard $\pi_{1}(M)$ spaces $(X, \mu)$. Here, $|M|^{L^{\infty}(X, \mu, \mathbb{R})}$ denotes the number defined like $|M|^{X}$, but using $L^{\infty}(X, \mu, \mathbb{R})$ instead of $L^{\infty}(X, \mu, \mathbb{Z})$.

We recall two "indecomposability" notions for parameter spaces from ergodic theory:

Definition 4.8 (ergodic/mixing parameter spaces). Let $\Gamma$ be a countable group. 
- A standard $\Gamma$-space $(X, \mu)$ is ergodic if every $\Gamma$-invariant measurable subset $A \subset X$ satisfies $\mu(A) \in\{0,1\}$ (equivalently, $L^{\infty}(X, \mu, \mathbb{Z})^{\Gamma}$ contains only the constant functions).

- A standard $\Gamma$-space $(X, \mu)$ is called mixing if for all measurable subsets $A, B \subset X$ and all sequences $\left(g_{n}\right)_{n \in \mathbb{N}}$ in $\Gamma$ with $\lim _{n \rightarrow \infty} g_{n}=\infty$ we have

$$
\lim _{n \rightarrow \infty} \mu\left(A \cap g_{n} \cdot B\right)=\mu(A) \cdot \mu(B) .
$$

Here, $\lim _{n \rightarrow \infty} g_{n}=\infty$ means that the sequence $\left(g_{n}\right)_{n \in \mathbb{N}}$ eventually leaves any finite subset of $\Gamma$, i.e., that for all finite subsets $F \subset \Gamma$ there is an $N \in \mathbb{N}$ such that for all $n \in \mathbb{N}_{\geq N}$ we have $g_{n} \in \Gamma \backslash F$.

Clearly, for infinite discrete groups, any mixing parameter space is also ergodic. Moreover, any countably infinite group admits an essentially free mixing parameter space:

Example 4.9 (Bernoulli shift). The Bernoulli shift of a countable group $\Gamma$ is the standard Borel space $\left(\{0,1\}^{\Gamma}, \bigotimes_{\Gamma}\left(1 / 2 \cdot \delta_{0}+1 / 2 \cdot \delta_{1}\right)\right.$, endowed with the translation action. If $\Gamma$ is infinite, this standard $\Gamma$-space is essentially free and mixing (and hence ergodic) [28, Lemma 3.37].

For ergodic parameter spaces, the parametrised fundamental class indeed is a generator of the corresponding top homology with twisted coefficients:

Remark 4.10. Let $M$ be an oriented closed connected $n$-manifold with fundamental group $\Gamma$, and let $(X, \mu)$ be an ergodic standard $\Gamma$-space. Then the inclusion $\mathbb{Z} \hookrightarrow L^{\infty}(X, \mathbb{Z})^{\Gamma}$ as constant functions is an isomorphism, and so the change of coefficients homomorphism

$$
H_{n}\left(i_{M}^{X}\right): \mathbb{Z} \cong H_{n}(M, \mathbb{Z}) \longrightarrow H_{n}\left(M, L^{\infty}(X, \mathbb{Z})\right) \cong L^{\infty}(X, \mathbb{Z})^{\Gamma} \cong \mathbb{Z}
$$

is an isomorphism; the isomorphism $H_{n}\left(M, L^{\infty}(X, \mathbb{Z})\right) \cong L^{\infty}(X, \mathbb{Z})^{\Gamma}$ is a consequence of Poincaré duality with twisted coefficients [33, Theorem 2.1, p. 23].

Furthermore, we will see that ergodic parameters suffice to describe the integral foliated simplicial volume (Proposition 4.17).

Remark 4.11 (lack of functoriality). Ordinary simplicial volume has the following functoriality property: If $f: M \longrightarrow N$ is a continuous map betweeen oriented closed connected manifolds of the same dimension of degree $d$, then

$$
|d| \cdot\|N\|=\left\|d \cdot[N]_{\mathbb{R}}\right\|_{1}=\left\|H_{*}(f, \mathbb{R})\left([M]_{\mathbb{R}}\right)\right\|_{1} \leq\|M\| .
$$

However, when dealing with integral coefficients, the first equality might fail in general (because we will not be able to divide representatives of $d \cdot[N]_{\mathbb{Z}}$ by $d$ ). Therefore, integral simplicial volume, stable integral simplicial volume and integral foliated simplicial volume suffer from a lack of good estimates in terms of mapping degrees.

In the following, we will investigate some of the effects of changing parameter spaces. To this end, we will use the following comparison mechanism:

Proposition 4.12 (comparing parameter spaces). Let $M$ be an oriented closed connected $n$-manifold with fundamental group $\Gamma$, let $(X, \mu)$ and $(Y, \nu)$ 
be standard $\Gamma$-spaces, and let $\varphi: X \longrightarrow Y$ be a measurable $\Gamma$-map. Moreover, suppose that

$$
\mu\left(\varphi^{-1}(A)\right) \leq \nu(A)
$$

holds for all measurable sets $A \subset Y$. Then

$$
|M|^{X} \leq|M|^{Y} .
$$

Proof. We consider the (well-defined) chain map $\Phi:=L^{\infty}(\varphi, \mathbb{Z}) \otimes_{\mathbb{Z} \Gamma} \operatorname{id}_{C_{*}(\widetilde{M}, \mathbb{Z})}$ :

$$
\begin{aligned}
\Phi: L^{\infty}(Y, \mathbb{Z}) \otimes_{\mathbb{Z} \Gamma} C_{*}(\widetilde{M}, \mathbb{Z}) & \longrightarrow L^{\infty}(X, \mathbb{Z}) \otimes_{\mathbb{Z} \Gamma} C_{*}(\widetilde{M}, \mathbb{Z}) \\
f \otimes \sigma & \longmapsto f \circ \varphi \otimes \sigma .
\end{aligned}
$$

In view of the compatibility of $\varphi$ with the measures (Equation (1)), we see that

$$
|\Phi(c)|^{X} \leq|c|^{Y}
$$

holds for all chains $c \in L^{\infty}(Y, \mathbb{Z}) \otimes_{\mathbb{Z} \Gamma} C_{*}(\widetilde{M}, \mathbb{Z})$. Moreover, Equation (1) shows that $\varphi$ is $\nu$-almost surjective. In particular, $L^{\infty}(\varphi, \mathbb{Z})$ maps $\nu$-almost constant functions to $\mu$-almost constant functions (with the same value). From this we can easily conclude that $\Phi$ maps $Y$-parametrised fundamental cycles to $X$-parametrised fundamental cycles. Taking the infimum over all $Y$-parametrised fundamental cycles of $M$ thus leads to $|M|^{X} \leq|M|^{Y}$.

We will now consider products, convex combinations, ergodic decomposition and induction/restriction of parameter spaces.

\subsection{Products of parameter spaces.}

Proposition 4.13 (products of parameter spaces). Let $M$ be an oriented closed connected $n$-manifold with fundamental group $\Gamma$.

(1) Let I be a non-empty, countable (or finite) set. If $\left(X_{i}, \mu_{i}\right)_{i \in I}$ is a family of standard $\Gamma$-spaces, then also the product

$$
(Z, \zeta):=\left(\prod_{i \in I} X_{i}, \bigotimes_{i \in I} \mu_{i}\right)
$$

equipped with the diagonal $\Gamma$-action, is a standard $\Gamma$-space, and

$$
|M|^{Z} \leq \inf _{i \in I}|M|^{X_{i}} .
$$

(2) Let $(X, \mu)$ be a standard $\Gamma$-space and let $(Y, \nu)$ be some standard Borel probability space. Then

$$
|M|^{Z}=|M|^{X},
$$

where $Z:=X \times Y$ is given the $\Gamma$-action induced by the $\Gamma$-action on $X$ and where $\zeta:=\mu \otimes \nu$ is the product measure on $Z$.

Proof. The first part follows by applying Proposition 4.12 for all $i \in I$ to the projection $\prod_{j \in I} X_{j} \longrightarrow X_{i}$.

We now show the second part (following a similar argument by Schmidt [28, Proposition 5.29]): We can view $(Y, \nu)$ as standard $\Gamma$-space with trivial $\Gamma$ action. Then we obtain

$$
|M|^{Z} \leq|M|^{X}
$$


from the first part. For the converse inequality, we consider a $Z$-parametrised fundamental cycle $c=\sum_{j=0}^{k} f_{j} \otimes \sigma_{j} \in L^{\infty}(Z, \mathbb{Z}) \otimes_{\mathbb{Z} \Gamma} C_{n}(\widetilde{M}, \mathbb{Z})$ in reduced form. So, if $c_{\mathbb{Z}} \in \mathbb{Z} \otimes_{\mathbb{Z} \Gamma} C_{n}(\widetilde{M}, \mathbb{Z})$ is a fundamental cycle of $M$, there is a chain $d \in L^{\infty}(Z, \mathbb{Z}) \otimes_{\mathbb{Z} \Gamma} C_{n+1}(\widetilde{M}, \mathbb{Z})$ such that

$$
c-c_{\mathbb{Z}}=\partial d \in L^{\infty}(Z, \mathbb{Z}) \otimes_{\mathbb{Z} \Gamma} C_{n}(\widetilde{M}, \mathbb{Z}) .
$$

Therefore, for $\nu$-almost all $y \in Y$, the chain

$$
c_{y}:=\sum_{j=0}^{k}\left(x \mapsto f_{j}(x, y)\right) \otimes \sigma_{j} \in L^{\infty}(X, \mathbb{Z}) \otimes_{\mathbb{Z} \Gamma} C_{n}(\widetilde{M}, \mathbb{Z})
$$

is well-defined ( $\Gamma$ acts trivially on $Y$ ) and an $X$-parametrised fundamental cycle in reduced form (witnessed by the corresponding evaluation of $d$ at $y$ ). By Fubini's theorem,

$$
\begin{aligned}
|c|^{Z} & =\int_{X \times Y} \sum_{j=0}^{k}\left|f_{j}\right| d(\mu \otimes \nu)=\int_{Y} \int_{X} \sum_{j=0}^{k}\left|f_{j}(x, y)\right| d \mu(x) d \nu(y) \\
& =\int_{Y}\left|c_{y}\right|^{X} d \nu(y) .
\end{aligned}
$$

Hence, there is a $y \in Y$ such that $c_{y}$ is an $X$-parametrised fundamental cycle and $\left|c_{y}\right|^{X} \leq|c|^{Z}$. Taking the infimum over all $Z$-parametrised fundamental cycles $c$ shows $|M|^{X} \leq|M|^{Z}$, as desired.

Taking products of parameter spaces hence shows that the infimum in the definition of integral foliated simplicial volume is a minimum:

Corollary 4.14. Let $M$ be an oriented closed connected manifold with fundamental group $\Gamma$. Then there exists a standard $\Gamma$-space $(X, \mu)$ with essentially free $\Gamma$-action satisfying

$$
|M|=|M|^{X} .
$$

Proof. Let $\left(X_{0}, \mu_{0}\right)$ be a standard $\Gamma$-space with essentially free $\Gamma$-action, e.g., the Bernoulli shift of $\Gamma$ (Example 4.9) (or, in the case of finite $\Gamma$ just $\Gamma$ with the normalised counting measure). For $n \in \mathbb{N}_{>0}$ let $\left(X_{n}, \mu_{n}\right)$ be a standard $\Gamma$-space with

$$
|M|^{X_{n}} \leq|M|+\frac{1}{n}
$$

Then the diagonal $\Gamma$-action on $(X, \mu):=\left(\prod_{n \in \mathbb{N}} X_{n}, \bigotimes_{n \in \mathbb{N}} \mu_{n}\right)$ is essentially free and we obtain $|M|^{X}=|M|$ from Proposition 4.13,

\subsection{Convex combinations of parameter spaces.}

Proposition 4.15 (convex combinations of parameter spaces). Let $M$ be an oriented closed connected $n$-manifold with fundamental group $\Gamma$, let $(X, \mu)$ and $(Y, \nu)$ be two standard $\Gamma$-spaces, and let $t \in[0,1]$. Then

$$
|M|^{Z}=t \cdot|M|^{X}+(1-t) \cdot|M|^{Y}
$$

where $Z:=X \sqcup Y$ is the disjoint union of $X$ and $Y$ endowed with the obvious $\Gamma$-action and the probability measure $\zeta:=t \cdot \mu \sqcup(1-t) \cdot \nu$. 
Proof. Under the mutually inverse $\mathbb{Z} \Gamma$-isomorphisms

$$
\begin{aligned}
L^{\infty}(Z, \zeta, \mathbb{Z}) & \longleftrightarrow L^{\infty}(X, \mu, \mathbb{Z}) \oplus L^{\infty}(Y, \nu, \mathbb{Z}) \\
f & \longmapsto\left(\left.f\right|_{X},\left.f\right|_{Y}\right) \\
\chi_{X} \cdot f+\chi_{Y} \cdot g & \longleftrightarrow(f, g)
\end{aligned}
$$

the constant function 1 on $Z$ corresponds to $(1,1)$, and for all $f \in L^{\infty}(Z, \zeta, \mathbb{Z})$ we have

$$
\int_{Z} f d \zeta=\left.t \cdot \int_{X} f\right|_{X} d \mu+\left.(1-t) \cdot \int_{Y} f\right|_{Y} d \nu
$$

Therefore, the same arguments as in Proposition 4.12 show that under the induced mutually inverse chain isomorphisms

$$
\begin{aligned}
L^{\infty}(Z, \zeta, \mathbb{Z}) \otimes_{\mathbb{Z} \Gamma} C_{n}(\widetilde{M}, \mathbb{Z}) \longleftrightarrow & L^{\infty}(X, \mu, \mathbb{Z}) \otimes_{\mathbb{Z} \Gamma} C_{n}(\widetilde{M}, \mathbb{Z}) \\
& \oplus L^{\infty}(Y, \nu, \mathbb{Z}) \otimes_{\mathbb{Z} \Gamma} C_{n}(\widetilde{M}, \mathbb{Z})
\end{aligned}
$$

$Z$-parametrised fundamental cycles correspond to pairs of $X$-parametrised and $Y$-parametrised fundamental cycles and that (by applying the arguments in both directions)

$$
\begin{aligned}
& |M|^{Z} \geq t \cdot|M|^{X}+(1-t) \cdot|M|^{Y}, \quad \text { and } \\
& |M|^{Z} \leq t \cdot|M|^{X}+(1-t) \cdot|M|^{Y} .
\end{aligned}
$$

In combination with Example 4.5 we obtain:

Corollary 4.16. Let $M$ be an oriented closed connected manifold with fundamental group $\Gamma$. Then

$$
\left\{|M|^{X} \mid(X, \mu) \text { is a standard } \Gamma \text {-space }\right\}=\left[|M|,\|M\|_{\mathbb{Z}}\right] \subset \mathbb{R} .
$$

4.4. Ergodic decomposition of parameter spaces. We will now show that ergodic parameter spaces suffice to describe the integral foliated simplicial volume:

Proposition 4.17 (ergodic parameters suffice). Let $M$ be an oriented closed connected manifold with fundamental group $\Gamma$.

(1) If $(X, \mu)$ is a standard $\Gamma$-space and $\varepsilon \in \mathbb{R}_{>0}$, then there is a $\Gamma$-invariant ergodic probability measure $\mu^{\prime}$ on the measurable $\Gamma$-space $X$ with

$$
|M|^{\left(X, \mu^{\prime}\right)} \leq|M|^{(X, \mu)}+\varepsilon .
$$

(2) In particular: For every $\varepsilon \in \mathbb{R}_{>0}$ there is an ergodic standard $\Gamma$ space $X$ with

$$
|M|^{X} \leq|M|+\varepsilon
$$

The proof of this proposition relies on the ergodic decomposition theorem:

Theorem 4.18 (ergodic decomposition [6, Theorem 5] [32, Theorem 4.2]). Let $\Gamma$ be a countable group and let $(X, \mu)$ be a standard $\Gamma$-space. Then there is a probability space $(P, \nu)$ and a familiy $\left(\mu_{p}\right)_{p \in P}$ of $\Gamma$-ergodic probability measures on the measurable $\Gamma$-space $X$ with the following property: For each Borel subset $A \subset X$, the function

$$
\begin{aligned}
P & \longrightarrow[0,1] \\
p & \longmapsto \mu_{p}(A)
\end{aligned}
$$


is measurable and

$$
\mu(A)=\int_{P} \mu_{p}(A) d \nu(p) .
$$

In view of this theorem all standard $\Gamma$-spaces can be seen as an assembly of ergodic $\Gamma$-spaces. However, we have to be careful about the sets of measure 0 with respect to the involved measures. Therefore, we consider the following "strict" function spaces and chain complexes:

Definition 4.19. Let $X$ be a measurable space. We write $B(X, \mathbb{Z})$ for the set of bounded measurable functions of type $X \longrightarrow \mathbb{Z}$. If $\mu$ is a measure on $X$, we write

$$
N(X, \mu, \mathbb{Z}):=\left\{f \in B(X, \mathbb{Z}) \mid \mu\left(f^{-1}(\mathbb{Z} \backslash 0)\right)=0\right\}
$$

for the set of all functions vanishing $\mu$-almost everywhere.

Remark 4.20. Let $M$ be an oriented closed connected manifold with fundamental group $\Gamma$ and let $(X, \mu)$ be a standard $\Gamma$-space. By definition,

$$
L^{\infty}((X, \mu), \mathbb{Z}) \cong B(X, \mathbb{Z}) / N(X, \mu, \mathbb{Z}),
$$

and this isomorphism of $\mathbb{Z} \Gamma$-modules gives rise to an isomorphism

$$
L^{\infty}((X, \mu), \mathbb{Z}) \otimes_{\mathbb{Z} \Gamma} C_{*}(\widetilde{M}, \mathbb{Z}) \cong \frac{B(X, \mathbb{Z}) \otimes_{\mathbb{Z} \Gamma} C_{*}(\widetilde{M}, \mathbb{Z})}{N(X, \mu, \mathbb{Z}) \otimes_{\mathbb{Z} \Gamma} C_{*}(\widetilde{M}, \mathbb{Z})}
$$

of chain complexes (because the chain modules of $C_{*}(\widetilde{M}, \mathbb{Z})$ are free, and hence flat, over $\mathbb{Z} \Gamma$ ).

Proof of Propostion 4.17. It suffices to prove the first part. Let $(X, \mu)$ be a standard $\Gamma$-space, let $\varepsilon \in \mathbb{R}_{>0}$, and let $n:=\operatorname{dim} M$. Then there is an $(X, \mu)$ parametrised fundamental cycle $c=\sum_{j=0}^{k} f_{j} \otimes \sigma_{j} \in L^{\infty}((X, \mu), \mathbb{Z}) \otimes_{\mathbb{Z} \Gamma}$ $C_{n}(\widetilde{M}, \mathbb{Z})$ with

$$
\sum_{j=0}^{k} \int_{X}\left|f_{j}\right| d \mu \leq|M|^{(X, \mu)}+\varepsilon
$$

Let $c_{\mathbb{Z}} \in \mathbb{Z} \otimes_{\mathbb{Z} \Gamma} C_{n}(\widetilde{M}, \mathbb{Z})$ be an integral fundamental cycle of $M$. Because $c$ is an $(X, \mu)$-parametrised fundamental cycle of $M$, we can find a chain $d \in L^{\infty}((X, \mu), \mathbb{Z}) \otimes_{\mathbb{Z} \Gamma} C_{n+1}(\widetilde{M}, \mathbb{Z})$ satisfying

$$
c-c_{\mathbb{Z}}=\partial d \in L^{\infty}((X, \mu), \mathbb{Z}) \otimes_{\mathbb{Z} \Gamma} C_{n}(\widetilde{M}, \mathbb{Z}) .
$$

In view of Remark 4.20, we can assume that the coefficients $f_{0}, \ldots, f_{k}$ of $c$ and those of $d$ lie in the "strict" function space $B(X, \mathbb{Z})$ and that there is a (without loss of generality, $\Gamma$-invariant) $\mu$-null set $A \subset X$ and a chain $c^{\prime} \in$ $B(X, \mathbb{Z}) \otimes_{\mathbb{Z} \Gamma} C_{n}(\widetilde{M}, \mathbb{Z})$ satisfying the relation

$$
c-c_{\mathbb{Z}}=\partial d+\chi_{A} \cdot c^{\prime} \in B(X, \mathbb{Z}) \otimes_{\mathbb{Z} \Gamma} C_{n}(\widetilde{M}, \mathbb{Z}) .
$$

Here, $\chi_{A} \cdot c^{\prime}$ uses the canonical $B(X, \mathbb{Z})^{\Gamma}-\mathbb{Z} \Gamma$-bimodule structure on $B(X, \mathbb{Z})$.

By the ergodic decomposition theorem (Theorem 4.18), we obtain a probability space $(P, \nu)$ and $\Gamma$-invariant ergodic probability measures $\left(\mu_{p}\right)_{p \in P}$ on $X$ with

$$
\mu(B)=\int_{X} \mu_{p}(B) d \nu(p)
$$


for all Borel sets $B \subset X$. Hence, for all $f \in B(X, \mathbb{Z})$ we have

$$
\int_{X} f d \mu=\int_{P} \int_{X} f d \mu_{p} d \nu(p)
$$

Taking $f:=\sum_{j=0}^{k}\left|f_{j}\right|$ and keeping in mind that $A$ is a $\mu$-null set, we thus find a $p \in P$ with

$$
\mu_{p}(A)=0 \text { and } \int_{X} f d \mu_{p} \leq \int_{X} f d \mu .
$$

We now show that $|M|^{\left(X, \mu_{p}\right)} \leq \int_{X} f d \mu \leq|M|^{(X, \mu)}+\varepsilon$ : To this end, we consider the chain

$$
c_{p}:=[c] \in \frac{B(X, \mathbb{Z}) \otimes_{\mathbb{Z} \Gamma} C_{n}(\widetilde{M}, \mathbb{Z})}{N\left(X, \mu_{p}, \mathbb{Z}\right) \otimes_{\mathbb{Z} \Gamma} C_{n}(\widetilde{M}, \mathbb{Z})} \cong L^{\infty}\left(\left(X, \mu_{p}\right), \mathbb{Z}\right) \otimes_{\mathbb{Z} \Gamma} C_{n}(\widetilde{M}, \mathbb{Z}) .
$$

Then $c_{p}$ is an $\left(X, \mu_{p}\right)$-parametrised fundamental cycle of $M$, because $c-c_{\mathbb{Z}}=$ $\partial d+\chi_{A} \cdot c^{\prime}$ holds in the "strict" twisted chain complex $B(X, \mathbb{Z}) \otimes_{\mathbb{Z} \Gamma} C_{*}(\widetilde{M}, \mathbb{Z})$ and $\mu_{p}(A)=0$, and so

$$
c_{p}-c_{\mathbb{Z}}=\partial[d] \in L^{\infty}\left(\left(X, \mu_{p}\right), \mathbb{Z}\right) \otimes_{\mathbb{Z} \Gamma} C_{n}(\widetilde{M}, \mathbb{Z}) .
$$

Furthermore, we obtain the desired estimate for the norms, namely

$$
\left.\left|c_{p} \mathbf{|}^{\left(X, \mu_{p}\right)} \leq \sum_{j=0}^{k} \int_{X}\right| f_{j}\left|d \mu_{p}=\int_{X} f d \mu_{p} \leq \int_{X} f d \mu \leq\right| M\right|^{(X, \mu)}+\varepsilon .
$$

As products of ergodic spaces are not necessarily ergodic, it is not clear that there is an analogue of Corollary 4.14 for ergodic parameter spaces:

Question 4.21. Is the integral foliated simplicial volume always given by an ergodic parameter space? Is the integral foliated simplicial volume always given by the Bernoulli shift of the fundamental group?

4.5. Integral foliated simplicial volume and finite coverings. We will now prove that integral foliated simplicial volume is multiplicative with respect to finite coverings:

Theorem 4.22 (multiplicativity of integral foliated simplicial volume). Let $M$ be an oriented closed connected $n$-manifold and let $p: N \longrightarrow M$ be a $d$-sheeted covering with $d \in \mathbb{N}_{>0}$. Then

$$
|M|=\frac{1}{d} \cdot|N| .
$$

The theorem will follow from compatibility with respect to restriction and induction of parameter spaces (Proposition 4.29 and 4.26).

Setup 4.23. Let $M$ be an oriented closed connected $n$-manifold with fundamental group $\Gamma$, and let $p: N \longrightarrow M$ be a $d$-sheeted covering with $d \in \mathbb{N}_{>0}$. Let $\Lambda$ be the fundamental group of $N$, and let $\Lambda^{\prime}=\pi_{1}(p)(\Lambda) \cong \Lambda$ be the subgroup of $\Gamma$ associated with $p$ (which has index $d$ in $\Gamma$ ). For notational simplicity, in the following, we will identify the groups $\Lambda$ and $\Lambda^{\prime}$ via the isomorphism given by $p$.

For the discussion of induction spaces and associated constructions, it will be necessary to choose representatives: 
Setup 4.24. Let $\Gamma$ be a countable group, let $\Lambda \subset \Gamma$ be a subgroup of finite index $d:=[\Gamma: \Lambda]$, and let $g_{1}, \ldots, g_{d} \in \Gamma$ be a set of representatives of $\Lambda$ in $\Gamma$ :

$$
\left\{g_{1} \cdot \Lambda, \ldots, g_{d} \cdot \Lambda\right\}=\Gamma / \Lambda .
$$

Definition 4.25 (induction). In the situation of Setup 4.24, let $(Y, \nu)$ be a standard $\Lambda$-space. Then the induction $\left(\Gamma \times_{\Lambda} Y, \mu\right)$ of $(Y, \nu)$ from $\Lambda$ to $\Gamma$ is the standard $\Gamma$-space defined as follows:

- The set

$$
\Gamma \times{ }_{\Lambda} Y:=\Gamma \times Y /\{(g \cdot h, y) \sim(g, h \cdot y) \mid g \in \Gamma, h \in \Lambda, y \in Y\}
$$

is endowed with the measurable structure induced from the bijection

$$
\begin{aligned}
\Gamma \times_{\Lambda} Y & \longrightarrow \Gamma / \Lambda \times Y \\
{\left[g_{j}, y\right] } & \longmapsto\left(g_{j} \cdot \Lambda, y\right)
\end{aligned}
$$

(where $\Gamma / \Lambda$ is given the discrete Borel structure). Moreover, the probability measure $\mu$ is the pull-back of the measure $1 / d \cdot \nu^{\prime} \otimes \nu$ on $\Gamma / \Lambda \times Y$ under this bijection, where $\nu^{\prime}$ denotes the counting measure on $\Gamma / \Lambda$.

- The $\Gamma$-action on $\Gamma \times_{\Lambda} Y$ is defined by

$$
\begin{aligned}
\Gamma \times\left(\Gamma \times_{\Lambda} Y\right) & \longrightarrow \Gamma \times_{\Lambda} Y \\
\left(g,\left[g^{\prime}, y\right]\right) & \longmapsto\left[g \cdot g^{\prime}, y\right] .
\end{aligned}
$$

Notice that in the above definition $\mu$ is indeed $\Gamma$-invariant, and that the measurable structure and the probability measure $\mu$ on the induction space do not depend on the chosen set of representatives.

Proposition 4.26 (induction of parameter spaces). In the situation of Setup 4.23, let $(Y, \nu)$ be a standard $\Lambda$-space. Then

$$
|M|^{\Gamma \times{ }_{\Lambda} Y}=\frac{1}{d} \cdot|N|^{Y}
$$

Proof. We choose representatives $g_{1}, \ldots, g_{d}$ for the index $d$ subgroup $\Lambda \subset \Gamma$ as in Setup 4.24. Induction of parameter spaces is compatible with algebraic induction of modules: We have (well-defined) mutually inverse $\mathbb{Z} \Gamma$ isomorphisms

$$
\begin{aligned}
\varphi: L^{\infty}\left(\Gamma \times_{\Lambda} Y, \mathbb{Z}\right) & \longrightarrow L^{\infty}(Y, \mathbb{Z}) \otimes_{\mathbb{Z} \Lambda} \mathbb{Z} \Gamma \\
f & \longmapsto \sum_{j=1}^{d}\left(y \mapsto f\left(\left[g_{j}, y\right]\right)\right) \otimes g_{j}, \\
\psi: L^{\infty}(Y, \mathbb{Z}) \otimes_{\mathbb{Z} \Lambda} \mathbb{Z} \Gamma & \longrightarrow L^{\infty}\left(\Gamma \times{ }_{\Lambda} Y, \mathbb{Z}\right) \\
f \otimes g_{j} & \longmapsto\left(\left[g_{k}, y\right] \mapsto\left\{\begin{array}{ll}
f(y) & \text { if } k=j \\
0 & \text { if } k \neq j
\end{array}\right) .\right.
\end{aligned}
$$

Because $p: M \longrightarrow N$ is a finite covering, $M$ and $N$ share the same universal covering space $\widetilde{M}=\widetilde{N}$ and the $\Lambda$-action on $\widetilde{N}$ is nothing but the restriction 
of the $\Gamma$-action on $\widetilde{M}$. Therefore, the above maps induce mutually inverse chain complex isomorphisms

$$
\begin{aligned}
\Phi: L^{\infty}\left(\Gamma \times_{\Lambda} Y, \mathbb{Z}\right) \otimes_{\mathbb{Z} \Gamma} C_{*}(\widetilde{M}, \mathbb{Z}) & \longrightarrow L^{\infty}(Y, \mathbb{Z}) \otimes_{\mathbb{Z} \Lambda} \mathbb{Z} \Gamma \otimes_{\mathbb{Z} \Gamma} C_{*}(\widetilde{M}, \mathbb{Z}) \\
& \cong L^{\infty}(Y, \mathbb{Z}) \otimes_{\mathbb{Z} \Lambda} C_{*}(\widetilde{N}, \mathbb{Z}) \\
f \otimes c & \longmapsto \sum_{j=1}^{d} f\left(\left[g_{j}, \cdot\right]\right) \otimes g_{j} \cdot c, \\
\Psi: L^{\infty}(Y, \mathbb{Z}) \otimes_{\mathbb{Z} \Lambda} C_{*}(\widetilde{N}, \mathbb{Z}) & \longrightarrow L^{\infty}\left(\Gamma \times_{\Lambda} Y, \mathbb{Z}\right) \otimes_{\mathbb{Z} \Gamma} C_{*}(\widetilde{M}, \mathbb{Z}) \\
f \otimes c & \longmapsto \psi(f \otimes 1) \otimes c .
\end{aligned}
$$

It is not difficult to see that $\Phi$ and $\Psi$ map $\Gamma \times_{\Lambda} Y$-parametrised fundamental cycles of $M$ to $Y$-parametrised fundamental cycles of $N$, and vice versa: It suffices to prove this claim for $\Phi$. For this, we use the following transfer type argument: Let $c_{\mathbb{Z}}=\sum_{j=1}^{k} a_{j} \otimes \sigma_{j} \in \mathbb{Z} \otimes_{\mathbb{Z} \Gamma} C_{n}(\widetilde{M}, \mathbb{Z}) \cong C_{n}(M, \mathbb{Z})$ be a fundamental cycle of $M$. By construction,

$$
\Phi \circ i_{M}^{\Gamma \times_{\Lambda} Y}\left(c_{\mathbb{Z}}\right)=i_{N}^{Y}\left(\sum_{j=1}^{k} a_{j} \otimes \sum_{\ell=1}^{d} g_{\ell} \cdot \sigma_{j}\right),
$$

and $g_{1} \cdot \sigma_{j}, \ldots, g_{d} \cdot \sigma_{j}$ are $\pi_{N}$-lifts of the $d$ different $p$-lifts of $\pi_{M} \circ \sigma_{j}$, where $\pi_{N}: \widetilde{N} \longrightarrow N$ and $\pi_{M}: \widetilde{M} \longrightarrow M$ denote the universal covering maps. Therefore, $\sum_{j=1}^{k} a_{j} \otimes \sum_{\ell=1}^{d} g_{\ell} \cdot \sigma_{j}$ is a fundamental cycle of $N$, which proves the claim about parametrised fundamental cycles.

By definition of the induction space (Definition 4.25), the $d$ copies of $Y$ inside $\Gamma \times{ }_{\Lambda} Y$ are each given the weight $1 / d$. Therefore, it is not difficult to show that

$$
|\Phi(c)|^{Y} \leq d \cdot|c|^{\Gamma \times{ }_{\Lambda} Y}
$$

holds for all chains $c \in L^{\infty}\left(\Gamma \times_{\Lambda} Y, \mathbb{Z}\right) \otimes_{\mathbb{Z} \Gamma} C_{*}(\widetilde{M}, \mathbb{Z})$, and that

$$
|\Psi(c)|^{\Gamma \times{ }_{\Lambda} Y} \leq \frac{1}{d} \cdot|c|^{Y}
$$

holds for all chains $c \in L^{\infty}(Y, \mathbb{Z}) \otimes_{\mathbb{Z} \Lambda} C_{*}(\tilde{N}, \mathbb{Z})$. Taking the infimum over all parametrised fundamental cycles therefore yields that

$$
|M|^{\Gamma \times{ }_{\Lambda} Y} \leq \frac{1}{d} \cdot|N|^{Y} \text { and }|N|^{Y} \leq d \cdot|M|^{\Gamma \times \times_{\Lambda} Y} .
$$

Corollary 4.27 (coset spaces as parameter space). In the situation of Setup 4.23 we have

$$
|M|^{\Gamma / \Lambda}=\frac{1}{d} \cdot\|N\|_{\mathbb{Z}}
$$

Here, we equip the finite set $\Gamma / \Lambda$ with the left $\Gamma$-action given by translation and the normalised counting measure.

Proof. Let $X$ be a standard $\Lambda$-space consisting of a single point. Then

$$
\Gamma / \Lambda \cong \Gamma \times{ }_{\Lambda} X
$$


(in the category of standard $\Gamma$-spaces). Hence, Proposition 4.26 and Example 4.5 show that

$$
|M|^{\Gamma / \Lambda}=\frac{1}{d} \cdot|N|^{X}=\frac{1}{d} \cdot\|N\|_{\mathbb{Z}} \cdot
$$

Conversely, we will now consider restriction of parameter spaces:

Definition 4.28 (restriction). Let $\Gamma$ be a group, let $(X, \mu)$ be a standard $\Gamma$-space, and let $\Lambda \subset \Gamma$ be a subgroup. Restricting the $\Gamma$-action on $X$ to $\Lambda$ (and keeping the same probability measure) results in a standard $\Lambda$-space, the restriction $\operatorname{res}_{\Lambda}^{\Gamma}(X, \mu)$ of $(X, \mu)$ from $\Gamma$ to $\Lambda$.

Proposition 4.29 (restriction of paramater spaces). In the situation of Setup 4.23 let $(X, \mu)$ be a standard $\Gamma$-space. Then

$$
\left.\frac{1}{d} \cdot|N|\right|^{\operatorname{res}_{\Lambda}^{\Gamma} X} \leq\left.|M|\right|^{X} .
$$

Proof. In view of Proposition 4.26, it suffices to show that

$$
|M|^{\Gamma \times{ }_{\Lambda} \operatorname{res}_{\Lambda}^{\Gamma} X} \leq|M|^{X} .
$$

The map

$$
\begin{aligned}
\Gamma \times_{\Lambda} \operatorname{res}_{\Lambda}^{\Gamma} X & \longrightarrow X \\
{[g, x] } & \longmapsto g \cdot x
\end{aligned}
$$

satisfies the hypotheses of Proposition 4.12, Therefore, we obtain the desired estimate $|M|^{\Gamma \times{ }_{\Lambda} \operatorname{res}_{\Lambda}^{\Gamma} X} \leq|M|^{X}$.

Example 4.30. In the situation of Proposition 4.29, in general, equality will not hold. For example, we could consider a double covering $S^{1} \longrightarrow S^{1}$ and a parameter space for the base manifold consisting of a single point.

We will now complete the proof of Theorem 4.22,

Proof of Theorem 4.22. From Proposition 4.29 we obtain, by taking the infimum over all standard $\Gamma$-spaces as parameter spaces for $M$,

$$
\frac{1}{d} \cdot|N| \leq|M|
$$

Conversely, from Proposition 4.26 we obtain, by taking the infimum over all standard $\Lambda$-spaces as parameter spaces for $N$,

$$
|M| \leq \frac{1}{d} \cdot|N|
$$

\section{A PROPORTIONALITY PRINCIPLE FOR INTEGRAL FOLIATED SIMPLICIAL VOLUME}

In this section, we will provide a proof of the proportionality principle Theorem 1.2 and of Corollary 1.3. We will use the language of measure equivalence of groups and techniques of Bader, Furman, and Sauer [1, Theorem 1.9]. 
5.1. Measure equivalence. The notion of measure equivalence was originally introduced by Gromov [14, 0.5.E1] as a measure-theoretic analogue of quasi-isometry.

Definition 5.1 (measure equivalence). Two countable groups $\Gamma$ and $\Lambda$ are called measure equivalent (ME) if there is a standard measure space $(\Omega, \mu)$ with commuting measure preserving $\Gamma$ - and $\Lambda$-actions, such that each of the actions admits a finite measure fundamental domain $X_{\Gamma}$ and $X_{\Lambda}$ respectively. The space $(\Omega, \mu)$ endowed with these actions is called an $M E$-coupling of $\Gamma$ and $\Lambda$. The ratio $c_{\Omega}=\mu\left(X_{\Lambda}\right) / \mu\left(X_{\Gamma}\right)$ is independent of the chosen fundamental domains and is called the coupling index of the ME-coupling $\Omega$.

In our context, the fundamental domains do not need to be strict fundamental domains; it suffices that $\Omega=\bigcup_{\gamma \in \Gamma} \gamma \cdot X_{\Gamma}$ is a disjoint decomposition up to measure 0. The cocycle in Definition 5.6 then will only be well-defined up to sets of measure 0; however, this poses no problems in the sequel as we will pass to $L^{1}$ - and $L^{\infty}$-spaces anyway.

Example 5.2 (lattices). A second countable locally compact group $G$ with its Haar measure is an ME-coupling for every pair of lattices $\Gamma$ and $\Lambda$ in $G$. Indeed, the Haar measure on $G$ is bi-invariant (because $G$ contains lattices), and the left actions

$$
\begin{aligned}
\Gamma \times G & \longrightarrow G & \Lambda \times G & \longrightarrow G \\
(\gamma, g) & \longmapsto \gamma g & (\lambda, g) & \longmapsto g \lambda^{-1}
\end{aligned}
$$

of $\Gamma$ and $\Lambda$ on $G$ given by multiplication in $G$ commute with each other.

Setup 5.3. Let $(\Omega, \mu)$ be an ME-coupling of $\Gamma$ and $\Lambda$. We suppose that both the actions are left actions, and we fix a fundamental domain for each of the two actions on the ME-coupling, denoted as $X_{\Gamma}$ and $X_{\Lambda}$ respectively.

Definition 5.4 (ergodic/mixing ME-coupling). In the situation of Setup 5.3. the ME-coupling is ergodic (resp. mixing) if the $\Gamma$-action on $\Lambda \backslash \Omega$ is ergodic (resp. mixing) and the $\Lambda$-action on $\Gamma \backslash \Omega$ is ergodic (resp. mixing).

Remark 5.5. Note that in this situation the $\Gamma$-action on $\Lambda \backslash \Omega$ is ergodic if and only if the $\Lambda$-action on $\Gamma \backslash \Omega$ is ergodic [12, Lemma 2.2].

Definition 5.6 (ME-cocycle). In the situation of the Setup 5.3, we define the measurable cocycle $\alpha_{\Lambda}$ associated to $X_{\Lambda}$ as the map

$$
\alpha_{\Lambda}: \Gamma \times X_{\Lambda} \rightarrow \Lambda
$$

such that $\alpha_{\Lambda}(\gamma, x)$ is the unique element satisfying $\gamma x \in \alpha_{\Lambda}(\gamma, x)^{-1} X_{\Lambda}$ for all $x \in X_{\Lambda}$ and $\gamma \in \Gamma$. Similarly, we define $\alpha_{\Gamma}: \Lambda \times X_{\Gamma} \rightarrow \Gamma$. If we choose another fundamental domain for $\Lambda \backslash \Omega$ then the associated cocycle is measurably cohomologous to $\alpha_{\Lambda}$ [12, Section 2] (the same for $\alpha_{\Gamma}$ ).

With this notation the natural left action of $\Gamma$ on $X_{\Lambda}$ and of $\Lambda$ on $X_{\Gamma}$ is described as follows:

$$
\begin{aligned}
\Gamma \times X_{\Lambda} & \longrightarrow X_{\Lambda} & \Lambda \times X_{\Gamma} & \longrightarrow X_{\Gamma} \\
(\gamma, x) & \longmapsto \gamma \bullet x:=\alpha_{\Lambda}(\gamma, x) \gamma x & (\lambda, y) & \longmapsto \lambda \bullet y:=\alpha_{\Gamma}(\lambda, y) \lambda y,
\end{aligned}
$$

where we write $\gamma \bullet x$ to distinguish it from the action $\gamma x$ of $\Gamma$ on $\Omega$. 
Remark 5.7. In the situation of the Setup 5.3, consider the standard $\Gamma$-space $\left(X_{\Lambda}, \mu_{\Lambda}=\mu\left(X_{\Lambda}\right)^{-1} \mu_{\left.\right|_{X_{\Lambda}}}\right)$ with $\Gamma$-action described above and the standard $\Gamma$-space $\Lambda \backslash \Omega$ with the probability measure induced from $\mu$ and the left translation $\Gamma$-action. Then the map $X_{\Lambda} \hookrightarrow \Omega \rightarrow \Lambda \backslash \Omega$ is a measure isomorphism.

Definition 5.8 (bounded ME-coupling). In the situation of the Setup 5.3, assume that $\Lambda$ is finitely generated, and let $l: \Lambda \rightarrow \mathbb{N}$ be the length function associated to some word-metric on $\Lambda$. We say that the fundamental domain $X_{\Lambda}$ is bounded if, for every $\gamma \in \Gamma$, the function $x \mapsto l\left(\alpha_{\Lambda}(\gamma, x)\right)$ is in $L^{\infty}\left(X_{\Lambda}, \mathbb{R}\right)$.

Let $\Gamma$ and $\Lambda$ be finitely generated. An ME-coupling of $\Gamma$ and $\Lambda$ is bounded if it admits bounded $\Gamma$ - and $\Lambda$-fundamental domains.

Example 5.9. A connected second countable locally compact group $G$ with its Haar measure is a bounded ME-coupling for every pair of uniform lattices in $G$ [2, p. 321] [30, Corollary 6.12 p. 58].

5.2. Homology of groups. In the aspherical case, we can express integral foliated simplicial volume in terms of group homology: Let $\Gamma$ be a discrete group. The bar resolution of $\Gamma$ is the $\mathbb{Z} \Gamma$-chain complex $C_{*}(\Gamma)$ defined as follows: for each $n \in \mathbb{N}$ let

$$
C_{n}(\Gamma)=\left\{\sum_{\gamma \in \Gamma^{n+1}} a_{\gamma} \cdot \gamma_{0} \cdot\left[\gamma_{1}|\cdots| \gamma_{n}\right] \mid \forall_{\gamma=\left(\gamma_{0}, \ldots, \gamma_{n}\right) \in \Gamma^{n+1}} a_{\gamma} \in \mathbb{Z}\right\}
$$

with the $\Gamma$-action characterized by

$$
\bar{\gamma} \cdot\left(\gamma_{0} \cdot\left[\gamma_{1}|\cdots| \gamma_{n}\right]\right)=\left(\bar{\gamma} \cdot \gamma_{0}\right) \cdot\left[\gamma_{1}|\cdots| \gamma_{n}\right]
$$

for all $\bar{\gamma} \in \Gamma$ and all $\gamma \in \Gamma^{n+1}$. The differential $\partial_{*}: C_{*}(\Gamma) \rightarrow C_{*-1}(\Gamma)$ is defined by

$$
\begin{aligned}
C_{n}(\Gamma) \longrightarrow & C_{n-1}(\Gamma) \\
\gamma_{0} \cdot\left[\gamma_{1}|\cdots| \gamma_{n}\right] \longmapsto & \gamma_{0} \cdot \gamma_{1} \cdot\left[\gamma_{2}|\cdots| \gamma_{n}\right] \\
& +\sum_{j=1}^{n-1}(-1)^{j} \cdot \gamma_{0} \cdot\left[\gamma_{1}|\cdots| \gamma_{j-1}\left|\gamma_{j} \cdot \gamma_{j+1}\right| \gamma_{j+2}|\cdots| \gamma_{n}\right] \\
& +(-1)^{n} \cdot \gamma_{0} \cdot\left[\gamma_{1}|\cdots| \gamma_{n-1}\right] .
\end{aligned}
$$

Moreover, the bar resolution is a normed chain complex (i.e., the differentials in each degree are bounded operators) with the $\ell^{1}$-norm given by

$$
\left\|\sum_{\gamma \in \Gamma^{n+1}} a_{\gamma} \cdot \gamma_{0} \cdot\left[\gamma_{1}|\cdots| \gamma_{n}\right]\right\|_{1}=\sum_{\gamma \in \Gamma^{n+1}}\left|a_{\gamma}\right| .
$$

We obtain a version of the bar resolution with coefficients using the tensor product: For every normed right $\mathbb{Z} \Gamma$-module $A$ let

$$
C_{*}(\Gamma, A):=A \otimes_{\mathbb{Z} \Gamma} C_{*}(\Gamma) .
$$

Definition 5.10 (group homology). Let $\Gamma$ be a discrete group and $A$ be a normed right $\mathbb{Z} \Gamma$-module. Then the group homology of $\Gamma$ with coefficients in $A$ is

$$
H_{*}(\Gamma, A):=H_{*}\left(C_{*}(\Gamma, A)\right) .
$$

The $\ell^{1}$-norm on the chain complex induces an $\ell^{1}$-semi-norm on the group homology. 
Proposition 5.11. Let $M$ be an aspherical manifold with universal covering $\widetilde{M}$, and fundamental group $\Gamma$. Let $A$ be a normed right $\mathbb{Z} \Gamma$-module. Then there exists a natural chain map

$$
c_{\Gamma}: C_{*}(\widetilde{M}, A) \longrightarrow C_{*}(\Gamma, A)
$$

that induces an isometric isomorphism

$$
c_{\Gamma}: H_{*}(M, A) \longrightarrow H_{*}(\Gamma, A) .
$$

Proof. It is not difficult to see that the classical mutually inverse $\mathbb{Z} \Gamma$-chain homotopy equivalences $C_{*}(\widetilde{M}, \mathbb{Z}) \longleftrightarrow C_{*}(\Gamma, \mathbb{Z})$, defined using a $\Gamma$-fundamental domain on $\widetilde{M}$, are norm non-increasing. This gives the desired isometric isomorphism in homology.

Corollary 5.12. Let $M, \Gamma$, and $(X, \mu)$ be as in Definition 4.1. If $M$ is aspherical, there exists an isometric isomorphism

$$
H_{*}\left(M, L^{\infty}(X, \mathbb{Z})\right) \stackrel{\cong}{\longrightarrow} H_{*}\left(\Gamma, L^{\infty}(X, \mathbb{Z})\right),
$$

with respect to the $\ell^{1}$-semi-norm induced by the $\ell^{1}$-norm on $L^{\infty}(X, \mathbb{Z})$.

Lemma 5.13. Let $\Gamma$ be a countable group and let $(X, \mu)$ be a standard $\Gamma$ space as in Definition 4.1 . The inclusion $L^{\infty}(X, \mathbb{Z}) \hookrightarrow L^{1}(X, \mathbb{Z})$ has dense image with respect to the $\ell^{1}$-norm and induces an isometric map

$$
H_{*}\left(\Gamma, L^{\infty}(X, \mathbb{Z})\right) \longrightarrow H_{*}\left(\Gamma, L^{1}(X, \mathbb{Z})\right) .
$$

Proof. Inclusions of dense subcomplexes induce isometric maps on homology [28, Lemma 2.9][17, Proposition 1.7] (the cited proofs also carry over to this integral setting).

Remark 5.14. Let $M, \Gamma$, and $(X, \mu)$ be as in Definition 4.1. If $M$ is aspherical, by Corollary 5.12 and Lemma 5.13 we deduce that the $X$-parametrised simplicial volume of $M$ can be computed via the $\ell^{1}$-semi-norm on the group homology $H_{*}\left(\Gamma, L^{1}(X, \mathbb{Z})\right)$.

\subsection{Proportionality principle, general case.}

Proof of Theorem 1.2. Let $n \in \mathbb{N}$. Let $\Gamma$ and $\Lambda$ be fundamental groups of oriented closed connected aspherical $n$-manifolds $M$ and $N$ with positive simplicial volume. Assume that $(\Omega, \mu)$ is an ergodic bounded ME-coupling of $\Gamma$ and $\Lambda$. Fix a bounded fundamental domain $X_{\Gamma} \subset \Omega$ (resp. $X_{\Lambda} \subset \Omega$ ) of the $\Gamma$-action on $\Omega$ (resp. of the $\Lambda$-action on $\Omega$ ) and let $\alpha_{\Gamma}$ (resp. $\alpha_{\Lambda}$ ) be the associated cocycle (Definition 5.6).

Using the cocycles associated with the ME-coupling we translate parametrised fundamental cycles of one manifold into parametrised fundamental cycles of the other manifold, while controlling the $\ell^{1}$-semi-norm. More precisely: consider the standard space $\left(X_{\Gamma}, \mu_{\Gamma}=\mu\left(X_{\Gamma}\right)^{-1} \mu_{\left.\right|_{X_{\Gamma}}}\right)$ with the $\Lambda$-action defined in Equation (21). By Remark 5.14 we use the $\ell^{1}$-norm on $L^{1}\left(X_{\Gamma}, \mathbb{Z}\right) \otimes_{\mathbb{Z} \Lambda} C_{*}(\Lambda, \mathbb{Z})$ to estimate the $X_{\Gamma}$-parametrised simplicial volume of $N$. Let $\Lambda^{*+1} \times X_{\Gamma}$ be endowed with the diagonal $\Lambda$-action and which carries the product of the counting measure and $\mu_{\Gamma}$. We identify $L^{1}\left(X_{\Gamma}, \mathbb{Z}\right) \otimes_{\mathbb{Z} \Lambda} C_{*}(\Lambda, \mathbb{Z})$ with $L^{1}\left(\Lambda^{*+1} \times X_{\Gamma}, \mathbb{Z}\right)_{\Lambda}^{\text {fin }}$, where the superscript "fin" indicates the submodule of functions $f: \Lambda^{*+1} \times X_{\Gamma} \rightarrow \mathbb{Z}$ with the 


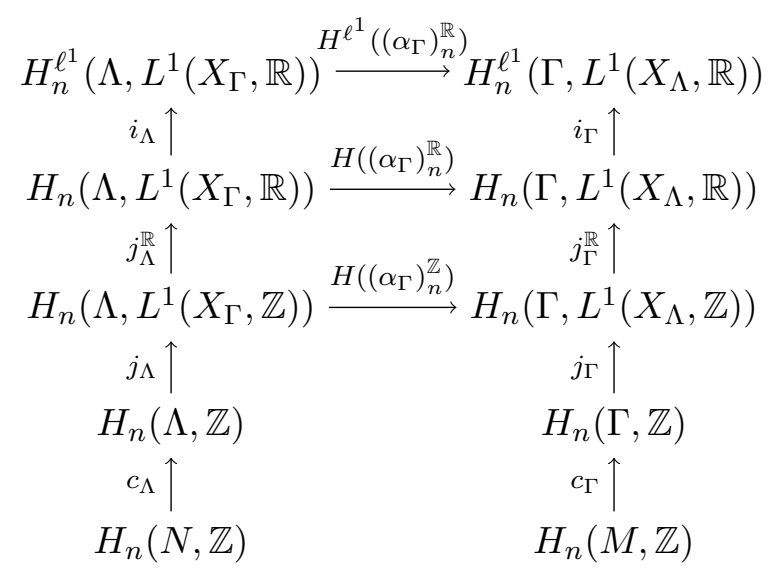

FiguRE 1. Effect of the cocycle in homology

property that there is a finite subset $F$ of $\Lambda^{*+1}$ such that $f$ is supported on $F \times X_{\Gamma}$, and where the subscript $\Lambda$ indicates the co-invariants.

Now, let us consider the measurable, countable-to-one, locally measure preserving (up to a constant factor) map [1, p. 284]

$$
\begin{aligned}
& \varphi_{n}^{\left(\alpha_{\Gamma}\right)}: \quad \Lambda^{n+1} \times X_{\Gamma} \longrightarrow \Gamma^{n+1} \times X_{\Lambda} \\
& \left(\lambda_{0}, \ldots, \lambda_{n}, y\right) \longmapsto\left(\alpha_{\Gamma}\left(\lambda_{0}^{-1}, y\right)^{-1}, \ldots, \alpha_{\Gamma}\left(\lambda_{n}^{-1}, y\right)^{-1}, \Lambda y \cap X_{\Lambda}\right)
\end{aligned}
$$

and define

$$
\begin{aligned}
\left(\alpha_{\Gamma}\right)_{n}^{\mathbb{Z}}: L^{1}\left(\Lambda^{n+1} \times X_{\Gamma}, \mathbb{Z}\right)_{\Lambda}^{\mathrm{fin}} \longrightarrow & L^{1}\left(\Gamma^{n+1} \times X_{\Lambda}, \mathbb{Z}\right)_{\Gamma}^{\mathrm{fin}} \\
f & \longmapsto \\
& (\gamma, x) \mapsto\left(\alpha_{\Gamma}\right)_{n}^{\mathbb{Z}}(f)(\gamma, x):= \\
& \sum_{(\lambda, y) \in\left(\varphi_{n}^{\left(\alpha_{\Gamma}\right)}\right)^{-1}(\gamma, x)} f(\lambda, y) .
\end{aligned}
$$

This map is the restriction to integral chains of the map described by Bader, Furman and Sauer [1, Theorem 5.7] up to rescaling by the coupling in$\operatorname{dex} c_{\Omega}=\mu\left(X_{\Lambda}\right) / \mu\left(X_{\Gamma}\right)$. The sum on the right hand side is a.e. finite 11, Lemma 5.8], and the boundedness of the coupling guarantees that $\left(\alpha_{\Gamma}\right)_{n}^{\mathbb{Z}}(f)$ has finite support whenever $f$ has finite support; moreover, a straightforward computation shows that the map is well-defined on the level of co-invariants. Hence, for a bounded ME-coupling of $\Gamma$ and $\Lambda,\left(\alpha_{\Gamma}\right)_{n}^{\mathbb{Z}}$ is a well-defined norm non-increasing (up to rescaling by the coupling index) chain map, and $\left(\alpha_{\Gamma}\right)_{n}^{\mathbb{Z}}$ induces a map

$$
H\left(\left(\alpha_{\Gamma}\right)_{n}^{\mathbb{Z}}\right): H_{n}\left(\Lambda, L^{1}\left(X_{\Gamma}, \mathbb{Z}\right)\right) \longrightarrow H_{n}\left(\Gamma, L^{1}\left(X_{\Lambda}, \mathbb{Z}\right)\right)
$$

of norm at most $1 / c_{\Omega}$.

Let us now consider the diagram in Figure 1, Since $M$ and $N$ are aspherical manifolds, Proposition 5.11 ensures that there exist isometric isomorphisms $c_{\Lambda}$ and $c_{\Gamma}$. The maps $j_{\Lambda}, j_{\Gamma}, j_{\Lambda}^{\mathbb{R}}, j_{\Gamma}^{\mathbb{R}}$ are the usual change of coefficients homomorphisms. In particular, by ergodicity of both actions, the maps $j_{\Lambda}$

\footnotetext{
${ }^{1}$ In Definition 4.1 we define a right action on $L^{\infty}(X, \mathbb{Z})\left(\right.$ and similarly on $L^{1}(X, \mathbb{Z})$ ) unlike op. cit., in which $L^{\infty}(X, \mathbb{Z})$ is endowed with a left action.
} 
and $j_{\Gamma}$ are (semi)-norm non-increasing isomorphisms. The inclusion of singular chains into $\ell^{1}$-chains induces the isometric homomorphisms $i_{\Lambda}$ and $i_{\Gamma}$. Finally $c_{\Omega} \cdot H^{\ell^{1}}\left(\left(\alpha_{\Gamma}\right)_{n}^{\mathbb{R}}\right)$ is the isometric isomorphism of Bader, Furman and Sauer [1, Theorem 5.7] defined between $\ell^{1}$-homology groups.

By the diagram in Figure 1 we have that

$$
H\left(\left(\alpha_{\Gamma}\right)_{n}^{\mathbb{R}}\right) \circ j_{\Lambda}^{\mathbb{R}} \circ j_{\Lambda} \circ c_{\Lambda}\left([N]_{\mathbb{Z}}\right)=m \cdot\left(j_{\Gamma}^{\mathbb{R}} \circ j_{\Gamma} \circ c_{\Gamma}\right)\left([M]_{\mathbb{Z}}\right)
$$

for some $m \in \mathbb{Z}$; here, we use that the $\Gamma$-action on $X_{\Lambda}$ is ergodic, and so $H_{n}\left(\Gamma, L^{1}\left(X_{\Lambda}, \mathbb{Z}\right)\right) \cong \mathbb{Z}$. By $\|N\|>0$ we have $\left\|i_{\Lambda} \circ j_{\Lambda}^{\mathbb{R}} \circ j_{\Lambda} \circ c_{\Lambda}\left([N]_{\mathbb{Z}}\right)\right\|_{1}>0$. Since $c_{\Omega} \cdot H^{\ell^{1}}\left(\left(\alpha_{\Gamma}\right)_{n}^{\mathbb{R}}\right)$ is an isometry we deduce that

$$
H^{\ell^{1}}\left(\left(\alpha_{\Gamma}\right)_{n}^{\mathbb{R}}\right) \circ i_{\Lambda} \circ j_{\Lambda}^{\mathbb{R}} \circ j_{\Lambda} \circ c_{\Lambda}\left([N]_{\mathbb{Z}}\right) \neq 0 .
$$

By the commutativity of the top square in Figure 1, we have $|m| \geq 1$ in Equation (3), and hence

$$
\begin{aligned}
\left\|\left(j_{\Gamma}^{\mathbb{R}} \circ j_{\Gamma} \circ c_{\Gamma}\right)\left([M]_{\mathbb{Z}}\right)\right\|_{1} & \leq|m| \cdot\left\|\left(j_{\Gamma}^{\mathbb{R}} \circ j_{\Gamma} \circ c_{\Gamma}\right)\left([M]_{\mathbb{Z}}\right)\right\|_{1} \\
& =\left\|H\left(\left(\alpha_{\Gamma}\right)_{n}^{\mathbb{R}}\right) \circ j_{\Lambda}^{\mathbb{R}} \circ j_{\Lambda} \circ c_{\Lambda}\left([N]_{\mathbb{Z}}\right)\right\|_{1} \\
& \leq c_{\Omega}^{-1} \cdot\left\|j_{\Lambda}^{\mathbb{R}} \circ j_{\Lambda} \circ c_{\Lambda}\left([N]_{\mathbb{Z}}\right)\right\|_{1} .
\end{aligned}
$$

By interchanging the roles of $\Gamma$ and $\Lambda$, we obtain the converse inequality and it turns out that $|m|=1$.

By the commutativity of the diagram in Figure 1 it follows:

$$
|M|^{X_{\Lambda}}=\left\|j_{\Gamma} \circ c_{\Gamma}\left([M]_{\mathbb{Z}}\right)\right\|_{1}=\left\|H\left(\left(\alpha_{\Gamma}\right)_{n}^{\mathbb{Z}}\right) \circ j_{\Lambda} \circ c_{\Lambda}\left([N]_{\mathbb{Z}}\right)\right\|_{1} \leq\left. c_{\Omega}^{-1} \cdot|N|\right|^{X_{\Gamma}},
$$

which implies

$$
|N|^{X_{\Gamma}} \geq c_{\Omega} \cdot|M|^{X_{\Lambda}} .
$$

Similarly we have the other inequality.

Since the map of Remark 5.7 induces an isometric isomorphism between $H_{n}\left(\Gamma, L^{1}\left(X_{\Lambda}, \mathbb{Z}\right)\right)$ and $H_{n}\left(\Gamma, L^{1}(\Lambda \backslash \Omega, \mathbb{Z})\right.$ ) (similarly for $\left.X_{\Gamma}\right)$, the previous equality does not depend on the choice of the fundamental domains:

$$
|N|^{\Gamma \backslash \Omega}=c_{\Omega} \cdot|M|^{\Lambda \backslash \Omega} .
$$

Let us now suppose that $(\Omega, \mu)$ is a mixing bounded ME-coupling of $\Gamma$ and $\Lambda$. Let $\left(X, \mu_{X}\right)$ be an ergodic standard $\Gamma$-space, with the structure of $\Lambda$-space with the trivial action. Then $\left(X \times \Omega, \mu_{X} \otimes \mu\right)$ is an ergodic bounded ME-coupling of $\Gamma$ and $\Lambda$ with respect to the left diagonal $\Gamma$ - and $\Lambda$-actions. Indeed if $F_{\Gamma}$ (resp. $F_{\Lambda}$ ) is a bounded fundamental domain for the action of $\Gamma$ on $\Omega($ resp. of $\Lambda)$ then $X_{\Gamma}=X \times F_{\Gamma}\left(\right.$ resp. $\left.X_{\Lambda}=X \times F_{\Lambda}\right)$ is a finite measure fundamental domain of the $\Gamma$-action on $X \times \Omega$ (resp. of the $\Lambda$-action). We can easily trace back the boundedness of $X_{\Gamma}$ (resp. $X_{\Lambda}$ ) to the one of $F_{\Gamma}$ (resp. $\left.F_{\Lambda}\right)$. Finally, by the property of mixing actions the $\Gamma$-action on $\Lambda \backslash(X \times \Omega) \cong X \times \Lambda \backslash \Omega$ is ergodic and $\left(X \times \Omega, \mu_{X} \otimes \mu\right)$ is an ergodic ME-coupling (see Definition 4.8 and Remark [5.5).

Applying now the previous construction to $\left(X \times \Omega, \mu_{X} \otimes \mu\right)$, we have

$$
|N|^{\Gamma \backslash(X \times \Omega)}=c_{X \times \Omega} \cdot|M|^{\Lambda \backslash(X \times \Omega)},
$$


where $c_{X \times \Omega}=\left(\mu_{X} \otimes \mu\right)\left(X \times X_{\Lambda}\right) /\left(\mu_{X} \otimes \mu\right)\left(X \times X_{\Gamma}\right)=\mu\left(X_{\Lambda}\right) / \mu\left(X_{\Gamma}\right)=c_{\Omega}$. Using Proposition 4.13 it follows that

$$
|N| \leq|N|^{\Gamma \backslash(X \times \Omega)}=c_{\Omega} \cdot|M|^{X \times(\Lambda \backslash \Omega)} \leq c_{\Omega} \cdot|M|^{X},
$$

and by Proposition 4.17 it turns out that

$$
|N| \leq c_{\Omega} \cdot|M| .
$$

By interchanging the roles of $\Gamma$ and $\Lambda$ we obtain the other inequality.

5.4. Proportionality principle, hyperbolic case. For the proof of Theorem 1.1 we need a proportionality principle for hyperbolic manifolds (Corollary 1.3$)$.

Proof of Corollary 1.3. Let $n \in \mathbb{N}$, let $\Gamma$ and $\Lambda$ be uniform lattices in $G:=$ Isom $^{+}\left(\mathbb{H}^{n}\right)$, and $\mathbb{H}^{n} / \Gamma$ and $\mathbb{H}^{n} / \Lambda$ be the associated oriented closed connected hyperbolic $n$-manifolds with fundamental groups $\Gamma$ and $\Lambda$. The group $G$ with its Haar measure $\mu$ is a bounded ME-coupling with respect to $\Gamma$ and $\Lambda$ (see Examples 5.2 and 5.9). The ergodicity of the coupling follows from the Moore Ergodicity Theorem [3, Theorem III.2.1]. Clearly, this coupling has coupling index $\operatorname{covol}(\Lambda) / \operatorname{covol}(\Gamma)$.

Notice that the standard $\Gamma$-space $\Lambda \backslash G$ (where $\Lambda$ acts on $G$ as in Example 5.2) is isomorphic to the coset space $G / \Lambda$ with the probability measure induced from the Haar measure $\mu$ and the left translation $\Gamma$-action; similarly, the standard $\Lambda$-space $\Gamma \backslash G$ with the $\Lambda$-action induced from Example 5.2 is isomorphic to the coset space $G / \Gamma$ with the left translation $\Lambda$-action.

Hence, Theorem 1.2 applied to this ergodic ME-coupling implies:

$$
\left|\mathbb{H}^{n} / \Lambda \mathbf{|}^{G / \Gamma}=\frac{\operatorname{covol}(\Lambda)}{\operatorname{covol}(\Gamma)} \cdot\right| \mathbb{H}^{n} /\left.\Gamma\right|^{G / \Lambda}
$$

Since the Moore Ergodicity Theorem [3, Theorem III.2.1] ensures also that $(G, \mu)$ is a mixing ME-coupling of $\Gamma$ and $\Lambda$, by the second part of Theorem 1.2 it turns out that

$$
\left|\mathbb{H}^{n} / \Lambda\right|=\frac{\operatorname{covol}(\Lambda)}{\operatorname{covol}(\Gamma)} \cdot\left|\mathbb{H}^{n} / \Gamma\right|
$$

\section{Comparing integral Foliated Simplicial VOlume AND STABLE INTEGRAL SIMPLICIAL VOLUME}

We will now compare integral foliated simplicial volume with stable integral simplicial volume. We start with a general sandwich estimate (Proposition 6.1), and we will then give more refined estimates for the hyperbolic case and for special parameter spaces. In particular, we will prove Corollary 1.4 and Theorem 1.5.

6.1. Sandwich estimate for integral foliated simplicial volume. Integral foliated simplicial volume interpolates between ordinary simplicial volume and stable integral simplicial volume:

Proposition 6.1 (comparing simplicial volumes). Let $M$ be an oriented closed connected manifold. Then

$$
\|M\| \leq|M| \leq\|M\|_{\mathbb{Z}}^{\infty} .
$$


Proof. The first inequality is contained in Proposition 4.6. The second inequality follows from Corollary 4.27 (alternatively: from Proposition 4.6 and Theorem 4.22).

Example 6.2 (integral foliated simplicial volume of surfaces). Let $M$ be an oriented closed connected surface of genus $g(M)$. Then

$$
|M|= \begin{cases}2 & \text { if } M \cong S^{2} \\ \|M\|=\|M\|_{\mathbb{Z}}^{\infty}=4 \cdot g(M)-4 & \text { otherwise. }\end{cases}
$$

Indeed, because $S^{2}$ is simply connected, $\left|S^{2}\right|=\left\|S^{2}\right\|_{\mathbb{Z}}=2$. If $M \not S^{2}$, then the classical computation of simplicial volume of aspherical surfaces [13, Section 0.2] shows that $\|M\|=\|M\|_{\mathbb{Z}}^{\infty}=4 \cdot g(M)-4$, and so the claim follows from Proposition 6.1

Hence, in the case of finite fundamental group, we have:

Corollary 6.3 (finite fundamental group). Let $M$ be an oriented closed connected manifold with finite fundamental group. Then

$$
|M|=\frac{1}{\left|\pi_{1}(M)\right|} \cdot\|\widetilde{M}\|_{\mathbb{Z}}=\|M\|_{\mathbb{Z}}^{\infty},
$$

where $\widetilde{M}$ is the universal covering of $M$. In contrast, $\|M\|=0$.

Proof. The universal covering $\widetilde{M} \longrightarrow M$ has $\left|\pi_{1}(M)\right|$ sheets; because $\pi_{1}(M)$ is finite, $\widetilde{M}$ indeed is an oriented closed connected manifold. Hence, Theorem 4.22 gives us

$$
|M|=\frac{1}{\left|\pi_{1}(M)\right|} \cdot|\widetilde{M}|
$$

Moreover, $|\widetilde{M}|=\|\widetilde{M}\|_{\mathbb{Z}}$ because $\widetilde{M}$ is simply connected (Example 4.5). So, in combination with Proposition 6.1, we obtain

$$
\|M\|_{\mathbb{Z}}^{\infty} \leq \frac{1}{\left|\pi_{1}(M)\right|} \cdot\|\widetilde{M}\|_{\mathbb{Z}}=|M| \leq\|M\|_{\mathbb{Z}}^{\infty} .
$$

On the other hand, simplicial volume of manifolds with finite (more generally, amenable) fundamental group is zero [13], and so $\|M\|=0$.

6.2. Proof of Corollary 1.4. We will now prove Corollary 1.4 with help of the proportionality principle for hyperbolic manifolds (Corollary 1.3). More precisely, we will prove the following, slightly more general, statement:

Theorem 6.4. Let $n \in \mathbb{N}$, and let $M$ and $N$ be oriented closed connected hyperbolic $n$-manifolds with fundamental groups $\Gamma$ and $\Lambda$, and let $G:=\operatorname{Isom}^{+}\left(\mathbb{H}^{n}\right)$. Let $S$ be a set of representatives of uniform lattices in $G$, containing a representative for every isometry class of oriented closed connected hyperbolic $n$-manifolds. The product $\prod_{\Lambda^{\prime} \in S} G / \Lambda^{\prime}$ is a standard $\Gamma$ space with respect to the diagonal translation action and the product of the probability measures induced by the (bi-invariant) Haar measure on $G$. Then

$$
|M| \leq|M| \prod_{\Lambda^{\prime} \in S} G / \Lambda^{\prime} \leq \frac{\operatorname{vol}(M)}{\operatorname{vol}(N)} \cdot\|N\|_{\mathbb{Z}}^{\infty} .
$$


Notice that in every dimension up to isometry there are only countably many different oriented closed connected hyperbolic manifolds. Hence, $S$ in the previous theorem is countable, and so the product $\prod_{\Lambda^{\prime} \in S} G / \Lambda^{\prime}$ indeed is a standard $\Gamma$-space.

Proof. We can view $\Gamma$ and $\Lambda$ as uniform lattices in $G=\operatorname{Isom}^{+}\left(\mathbb{H}^{n}\right)$ and we have $M=\mathbb{H}^{n} / \Gamma$ and $\operatorname{covol}(\Gamma)=\operatorname{vol}(M)$ (and similarly for $N$ and its finite coverings).

Let $N^{\prime} \longrightarrow N$ be a finite covering of $N$, let $\Lambda^{\prime}$ be the fundamental group of $N^{\prime}$, and let $d:=\left[\Lambda: \Lambda^{\prime}\right]$ be the number of sheets of this covering. From the proportionality principle (Corollary 1.3), Proposition 4.13, and Proposition 4.6 we obtain

$$
\begin{aligned}
|M| & =\left|\mathbb{H}^{n} / \Gamma\right| \leq\left|\mathbb{H}^{n} / \Gamma\right|^{\prod_{\Lambda^{\prime \prime} \in S} G / \Lambda^{\prime \prime}} \leq\left|\mathbb{H}^{n} / \Gamma\right|^{G / \Lambda^{\prime}} \\
& =\frac{\operatorname{covol}(\Gamma)}{\operatorname{covol}\left(\Lambda^{\prime}\right)} \cdot\left|\mathbb{H}^{n} / \Lambda^{\prime}\right|^{G / \Gamma}=\frac{\operatorname{covol}(\Gamma)}{\operatorname{covol}(\Lambda)} \cdot \frac{1}{\left[\Lambda: \Lambda^{\prime}\right]} \cdot\left|N^{\prime}\right|^{G / \Gamma} \\
& \leq \frac{\operatorname{vol}(M)}{\operatorname{vol}(N)} \cdot \frac{1}{d} \cdot\left\|N^{\prime}\right\|_{\mathbb{Z}} \cdot
\end{aligned}
$$

Taking the infimum over all finite coverings of $N$ finishes the proof.

This concludes the proof of Corollary 1.4.

\subsection{Proof of Theorem $\mathbf{1 . 5}$.}

Definition 6.5. Let $\Gamma$ be a finitely generated group and let $S$ be a set of finite index subgroups of $\Gamma$ (hence, $S$ is countable). Then the standard $\Gamma$-space $X_{\Gamma, S}$ is given by the set

$$
\prod_{\Lambda \in S} \Gamma / \Lambda
$$

equipped with the product measure of the normalised counting measures and the diagonal left $\Gamma$-action given by translating cosets.

Notice that in the situation of Definition 6.5 the $\Gamma$-action on $X_{\Gamma, S}$ is free if and only if $\bigcap_{\Lambda \in S} \Lambda=\{e\}$, and that the $\Gamma$-action is not ergodic in general.

Theorem 6.6 (products of coset spaces as parameter space). Let $M$ be an oriented closed connected manifold with fundamental group $\Gamma$, let $S$ be a set of finite index subgroups of $\Gamma$ that is stable under finite intersections. For $\Lambda \in S$ we denote the covering space of $M$ associated with the subgroup $\Lambda \subset \Gamma$ by $M_{\Lambda}$.

(1) Then

$$
|M|^{X_{\Gamma, S}}=\inf _{\Lambda \in S} \frac{1}{[\Gamma: \Lambda]} \cdot\left\|M_{\Lambda}\right\|_{\mathbb{Z}}
$$

(2) In particular: If $S$ is the set of all finite index subgroups of $\Gamma$, then

$$
|M|^{X_{\Gamma, S}}=\|M\|_{\mathbb{Z}}^{\infty} .
$$

The second part is nothing but Theorem 1.5 , 
Proof. By definition of the stable integral simplicial volume and because finite intersections of finite index subgroups of $\Gamma$ have finite index in $\Gamma$, it suffices to prove the first part.

We first show that the right hand side is an upper bound for the left hand side: For all $\Lambda \in S$ we have

$$
|M|^{X_{\Gamma, S}} \leq|M|^{\Gamma / \Lambda}=\frac{1}{[\Gamma: \Lambda]} \cdot\left\|M_{\Lambda}\right\|_{\mathbb{Z}}
$$

by Proposition 4.13 and Corollary 4.27, Taking the infimum yields

$$
|M|^{X_{\Gamma, S}} \leq \inf _{\Lambda \in S} \frac{1}{[\Gamma: \Lambda]} \cdot\left\|M_{\Lambda}\right\|_{\mathbb{Z}}
$$

It remains to show that the left hand side also is an upper bound for the right hand side: The main idea is to reduce the parameter space $X_{\Gamma, S}$ to finite products of coset spaces. So, let $F(S)$ be the set of finite subsets of $S$. For $F \in F(S)$ we write $\sigma_{F}$ for the $\sigma$-algebra of the finite product $X_{\Gamma, F}$ (which is just the power set of $X_{\Gamma, F}$ ), we write

$$
\pi_{F}: X_{\Gamma, S} \longrightarrow X_{\Gamma, F}
$$

for the canonical projection, and we define

$$
L_{F}:=\left\{f \circ \pi_{F} \mid f \in L^{\infty}\left(X_{\Gamma, F}, \mathbb{Z}\right)\right\} \subset L^{\infty}\left(X_{\Gamma, S}, \mathbb{Z}\right) .
$$

As first step, we will determine $|M|^{X_{\Gamma, F}}$ for $F \in F(S)$ : For all $\gamma, \gamma^{\prime} \in \Gamma$ we have

$$
\left(\forall_{\Lambda \in F} \quad \gamma \cdot \Lambda=\gamma^{\prime} \cdot \Lambda\right) \Longleftrightarrow \gamma \cdot \bigcap_{\Lambda \in F} \Lambda=\gamma^{\prime} \cdot \bigcap_{\Lambda \in F} \Lambda .
$$

Hence, $X_{\Gamma, F}=\prod_{\Lambda \in F} \Gamma / \Lambda$ is, as a $\Gamma$-parameter space, a finite convex combination of multiple copies of the coset space $\Gamma / \bigcap_{\Lambda \in F} \Lambda$. In view of Proposition 4.15 we obtain

$$
|M|^{X_{\Gamma, F}}=|M|^{\Gamma / \cap_{\Lambda \in F} \Lambda}
$$

As second step, we will now show that

$$
L:=\bigcup_{F \in F(S)} L_{F} \subset L^{\infty}\left(X_{\Gamma, S}, \mathbb{Z}\right)
$$

is $\|\cdot\|_{1}$-dense in $L^{\infty}\left(X_{\Gamma, S}, \mathbb{Z}\right)$ : The $\sigma$-algebra $\sigma$ on the product space $X_{\Gamma, S}$ is the product $\sigma$-algebra of the power sets of all factors $\Gamma / \Lambda$ with $\Lambda \in S$; i.e., $\sigma$ is generated by $\bigcup_{F \in F(S)} \pi_{F}^{-1}\left(\sigma_{F}\right)$. We now consider the system

$$
\sigma^{\prime}:=\left\{A \in \sigma \mid \chi_{A} \in \bar{L}^{\|\cdot\|_{1}}\right\}
$$

of subsets of $X_{\Gamma, S}$. In order to show that $L$ is $\|\cdot\|_{1}$-dense in $L^{\infty}\left(X_{\Gamma, S}, \mathbb{Z}\right)$ it suffices to prove that $\sigma^{\prime}=\sigma$ : It is easy to check that $\sigma^{\prime}$ is a $\sigma$-algebra on $X_{\Gamma, S}$. Moreover, by definition, $\bigcup_{F \in F(S)} \pi_{F}^{-1}\left(\sigma_{F}\right) \subset \sigma^{\prime}$. Hence, $\sigma^{\prime}=\sigma$, and so $L$ is $\|\cdot\|_{1}$-dense in $L^{\infty}\left(X_{\Gamma, S}, \mathbb{Z}\right)$.

Therefore, the map

$$
H_{*}(M, L) \longrightarrow H_{*}\left(M, L^{\infty}\left(X_{\Gamma, S}, \mathbb{Z}\right)\right)
$$

induced by the inclusion $L \hookrightarrow L^{\infty}\left(X_{\Gamma, S}, \mathbb{Z}\right)$ of coefficient $\mathbb{Z} \Gamma$-modules is isometric [28, Lemma 2.9] [17, Proposition 1.7] (the cited proofs carry over to this integral setting) and the $X_{\Gamma, S}$-fundamental class of $M$ is contained in 
the image; notice that the union $L=\bigcup_{F \in F(S)} L_{F}$ indeed is a $\mathbb{Z} \Gamma$-submodule of $L^{\infty}\left(X_{\Gamma, S}, \mathbb{Z}\right)$.

Let $c \in C_{*}(M, L)$ be an $L$-fundamental cycle of $M$. By definition of $L$, there exists an $F \in F(S)$ such that $c \in C_{*}\left(M ; L_{F}\right)$. Thus,

$$
|c|^{L} \geq|M|^{X_{\Gamma, F}}=|M|^{\Gamma / \bigcap_{\Lambda \in F} \Lambda} \geq \inf _{\Lambda \in S}|M|^{\Gamma / \Lambda}=\inf _{\Lambda \in S} \frac{1}{[\Gamma: \Lambda]} \cdot\left\|M_{\Lambda}\right\|_{\mathbb{Z}}
$$

the first inequality is a consequence of the isometric isomorphism $L_{F} \longrightarrow$ $L^{\infty}\left(X_{\Gamma, F}, \mathbb{Z}\right)$ induced from the projection $\pi_{F}$, the second equality was shown in the first step, the third inequality holds because $S$ is assumed to be closed under finite intersections, and the last equality follows from Corollary 4.27. Taking the infimum over all fundamental cycles and taking the isometry $H_{*}(M ; L) \longrightarrow H_{*}\left(M ; L^{\infty}\left(X_{\Gamma, S}, \mathbb{Z}\right)\right)$ into account gives the desired estimate

$$
|M|^{X_{\Gamma, S}} \geq \inf _{\Lambda \in S} \frac{1}{[\Gamma: \Lambda]} \cdot\left\|M_{\Lambda}\right\|_{\mathbb{Z}}
$$

Remark 6.7 (inverse limits). The proof of Theorem 6.6 carries over to the following modification: Instead of the product $\prod_{\Lambda \in S} \Gamma / \Lambda$ we can also consider the inverse limit $I_{\Gamma, S}$ of the system $(\Gamma / \Lambda)_{\Lambda \in S}$ with respect to the canonical projection maps between coset spaces of nested subgroups; we equip $I_{\Gamma, S}$ with the $\Gamma$-action induced from the translation action on the coset spaces and we equip $I_{\Gamma, S}$ with the Borel probability space structure given by the discrete $\sigma$-algebras on the coset spaces and the compatible system of normalised counting measures on the coset spaces. We then obtain

$$
|M|^{I_{\Gamma, S}}=\inf _{\Lambda \in S} \frac{1}{[\Gamma: \Lambda]} \cdot\left\|M_{\Lambda}\right\|_{\mathbb{Z}}
$$

While this inverse limit space might be harder to visualise than the product space $X_{\Gamma, S}$, it does have the advantage that it is ergodic.

\section{INTEGRAL FOLIATED SIMPLICIAL VOLUME OF HYPERBOLIC 3-MANIFOLDS}

We will now complete the proof of Theorem 1.1 by proving the following, more explicit, version:

Theorem 7.1 (integral foliated simplicial volume of hyperbolic 3-manifolds). Let $M$ be an oriented closed connected hyperbolic 3-manifold, and let $S$ be a set of representatives of uniform lattices in $G:=\operatorname{Isom}^{+}\left(\mathbb{H}^{3}\right)$ containing a representative for every isometry class of oriented closed connected hyperbolic 3-manifolds (see Theorem 6.4). Then

$$
|M|=|M|^{\prod_{\Lambda \in S} G / \Lambda}=\|M\| .
$$

Proof. Let $M$ be an oriented closed connected hyperbolic 3-manifold. In view of Theorem 1.6 there exists a sequence $\left(M_{n}\right)_{n \in \mathbb{N}}$ of oriented closed connected hyperbolic 3-manifolds with

$$
\lim _{n \rightarrow \infty} \frac{\left\|M_{n}\right\|_{\mathbb{Z}}^{\infty}}{\left\|M_{n}\right\|}=1
$$


By Corollary 1.4 (and Theorem 6.4, for the concrete parameter space), for all $n \in \mathbb{N}$ we have

$$
\|M\| \leq|M| \leq|M| \prod_{\Lambda \in S} G / \Lambda \leq \frac{\operatorname{vol} M}{\operatorname{vol} M_{n}} \cdot\left\|M_{n}\right\|_{\mathbb{Z}}^{\infty} .
$$

On the other hand, by the classical proportionality principle for simplicial volume for hyperbolic manifolds we have $\left\|M_{n}\right\|>0$ and

$$
\frac{\operatorname{vol} M}{\operatorname{vol} M_{n}}=\frac{\|M\|}{\left\|M_{n}\right\|}
$$

and so

$$
\|M\| \leq|M| \leq|M|^{\prod_{\Lambda \in S} G / \Lambda} \leq \frac{\|M\|}{\left\|M_{n}\right\|} \cdot\left\|M_{n}\right\|_{\mathbb{Z}}^{\infty} .
$$

Because of $\lim _{n \rightarrow \infty}\left\|M_{n}\right\|_{\mathbb{Z}}^{\infty} /\left\|M_{n}\right\|=1$ the right hand side converges to $\|M\|$ for $n \rightarrow \infty$. Hence, $\|M\|=|M|^{\prod_{\Lambda \in S} G / \Lambda}=|M|$, as desired.

Similarly to Francaviglia, Frigerio, and Martelli [8, Question 6.4], we hence ask:

Question 7.2. Does the integral foliated simplicial volume of oriented closed connected hyperbolic manifolds of dimension bigger than 3 also coincide with the simplicial volume? Does this even hold for all oriented closed connected aspherical manifolds?

\section{Stable integral Simplicial VOlume AND integral Foliated SIMPLICIAL VOLUME OF SEIFERT 3-MANIFOLDS}

For the sake of completeness, we add also the computation of stable integral simplicial volume and integral foliated simplicial volume of Seifert 3-manifolds:

Proposition 8.1 (Seifert case). Let $M$ be an oriented compact connected Seifert manifold with $\left|\pi_{1}(M)\right|=\infty$. Then $\|M\|_{\mathbb{Z}}^{\infty}=0$.

Proof. We follow step by step the related argument of Francaviglia, Frigerio, and Martelli [8, Proposition 5.11]. A Seifert manifold has a finite covering that is an $S^{1}$-bundle over an orientable surface $\Sigma$ with some Euler number $e \geq 0$. If the manifold $M$ has boundary, then $e=0$ and the bundle is a product $S^{1} \times \Sigma$. Since $S^{1} \times \Sigma$ covers itself with arbitrarily high degree, $S^{1} \times \Sigma$ (and hence also $M$ ) clearly has stable integral simplicial volume 0 .

If $M$ is closed, we denote the covering mentioned above by $(\Sigma, e)$. A closed connected orientable $S^{1}$-bundle over an orientable surface either is irreducible or $S^{1} \times S^{2}$ or $\mathbb{R} \mathbb{P}^{3} \# \mathbb{R P}^{3}$. Since $\mathbb{R} \mathbb{P}^{3} \# \mathbb{R} \mathbb{P}^{3}$ admits a double selfcovering, in this case the stable integral simplicial volume vanishes. Moreover, we have already considered the case $S^{1} \times \Sigma$. Therefore we restrict to irreducible $S^{1}$-bundles. By assumption, $(\Sigma, e)$ is a closed connected orientable irreducible Seifert manifold with $\left|\pi_{1}((\Sigma, e))\right|=\infty$. Thus, by Remark 3.4, the complexity is equal to the special complexity and it has the following bound [22]:

$$
c_{S}(\Sigma, e) \leq e+6 \cdot \chi_{-}(\Sigma)+6
$$

where $\chi_{-}(\Sigma)=\max \{-\chi(\Sigma), 0\}$. 
Let now $T$ be a minimal triangulation of $(\Sigma, e)$, and let $T^{\prime}$ be its first barycentric subdivision. Then $T^{\prime}$ is a semi-simplicial triangulation that allows us to define an integral fundamental cycle. Since the special complexity of $(\Sigma, e)$ is equal to the minimal number of tetrahedra in a triangulation and the barycentric subdivision of $\Delta^{3}$ consists of 24 tetrahedra, we have

$$
\|(\Sigma, e)\|_{\mathbb{Z}} \leq 24 \cdot c_{S}(\Sigma, e) \leq 24 \cdot\left(e+6 \cdot \chi_{-}(\Sigma)+6\right) .
$$

Now for every $d \in \mathbb{N}$ we construct a degree- $d^{2}$ covering $(\bar{\Sigma}, e) \longrightarrow(\Sigma, e)$ where $\bar{\Sigma}$ is a $d$-sheeted covering space of $\Sigma$ as in the argument of Francaviglia, Frigerio, and Martelli [8, Proposition 5.11] in order to conclude.

In particular, we obtain:

Corollary 8.2. Let $M$ be an oriented closed connected Seifert manifold with $\left|\pi_{1}(M)\right|=\infty$. Then

$$
\|M\|=|M|=\|M\|_{\mathbb{Z}}^{\infty}=0 .
$$

By Perelman's result a closed Seifert manifold with finite fundamental group admits an elliptic structure (i.e., it is a quotient of $S^{3}$ by a finite subgroup of $\mathrm{SO}(4)$ acting by rotations).

Proposition 8.3. Let $n \in \mathbb{N}_{>0}$, and let $M$ be an oriented closed connected elliptic $n$-manifold. Then

$$
|M|=\|M\|_{\mathbb{Z}}^{\infty}=\frac{1}{\left|\pi_{1}(M)\right|} \cdot \begin{cases}1 & \text { if } n \text { is odd } \\ 2 & \text { if } n \text { is even } .\end{cases}
$$

In contrast, $\|M\|=0$.

Proof. Corollary 6.3 shows that

$$
|M|=\|M\|_{\mathbb{Z}}^{\infty}=\frac{1}{\left|\pi_{1}(M)\right|} \cdot\left\|S^{n}\right\|_{\mathbb{Z}} .
$$

Clearly, $\left\|S^{n}\right\|_{\mathbb{Z}}=1$ if $n$ is odd, and $\left\|S^{n}\right\|_{\mathbb{Z}}=2$ if $n$ is even.

On the other hand,the simplicial volume of an elliptic manifold is always zero because simplicial volume is multiplicative under finite coverings [18, Proposition 4.1] and $\left\|S^{n}\right\|=0$ for all $n \in \mathbb{N}_{>0}$.

\section{REFERENCES}

[1] U. Bader, A. Furman, R. Sauer. Efficient subdivision in hyperbolic groups and applications. Groups, Geometry, and Dynamics, 7, pp. 263-292, 2013. Cited on page: 2 $1 8 \longdiv { 2 2 } 2 3$

[2] U. Bader, A. Furman, R. Sauer. Integrable measure equivalence and rigidity of hyperbolic lattices. Invent. Math., 194, pp. 313-379. Cited on page: 20

[3] M. B. Bekka, M. Mayer. Ergodic theory and topological dynamics of group actions on homogeneous spaces. London Mathematical Society Lecture Note Series, 269, Cambridge University Press, Cambridge, 2000. Cited on page: 24

[4] M. Bucher-Karlsson. The proportionality constant for the simplicial volume of locally symmetric spaces. Colloq. Math., 111(2), pp. 183-198, 2008. Cited on page: 2

[5] M. Bucher, M. Burger, A. Iozzi. A dual interpretation of the Gromov-Thurston proof of Mostow rigidity and volume rigidity for representations of hyperbolic lattices. Trends in Harmonic Analysis, Indam-Springer Series 3, Springer-Verlag, Italia, 2013. Cited on page: 2 60 
[6] R. H. Farrell. Representation of invariant measures. Illinois J. Math., 6, p. 447-467, 1962. Cited on page: 13

[7] S. Francaviglia. Hyperbolic volume of representations of fundamental groups of cusped 3-manifolds. Int. Math. Res. Not., 9, pp. 425-459, 2004. Cited on page: 26

[8] S. Francaviglia, R. Frigerio, B. Martelli. Stable complexity and simplicial volume of manifolds. Journal of Topology, 5, pp. 977-1010, 2012. Cited on page: 3 56 6 29 , 30

[9] R. Frigerio. (Bounded) continuous cohomology and Gromov's proportionality principle. Manuscripta Math., 134, pp. 435474, 2011. Cited on page: 2

[10] R. Frigerio, C. Pagliantini. The simplicial volume of hyperbolic manifolds with geodesic boundary. Algebr. Geom. Topol., 10, pp. 979-1001, 2010. Cited on page: 2 6 . 7

[11] K. Fujiwara, J. K. Manning, Simplicial volume and fillings of hyperbolic manifolds. Algebr. Geom. Topol., 11, pp. 2237-2264, 2011. Cited on page: 2 6

[12] A. Furman. Gromov's measure equivalence and rigidity of higher rank lattices. Ann. of Math., 150, no.3, pp. 1059-1081, 1999. Cited on page: 19

[13] M. Gromov. Volume and bounded cohomology. Inst. Hautes Études Sci. Publ. Math., 56, pp. 5-99, 1983. Cited on page: 10 10 6 25

[14] M. Gromov. Asymptotic invariants of infinite groups. Geometric group theory, Vol. 2 (Sussex 1991). London Math. Soc. Lectures Notes Ser., 182, Cambridge Univ. Press, Cambridge, pp. 1-295, 1993. Cited on page: 10 19.

[15] M. Gromov. Metric structures for Riemannian and non-Riemannian spaces. With appendices by M. Katz, P. Pansu, and S. Semmes, translated by S.M. Bates. Progress in Mathematics, 152, Birkhäuser, 1999. Cited on page: 1

[16] A. Kechris. Classical Descriptive Set Theory, Graduate Texts in Mathematics, 156. Springer, 1995. Cited on page:

[17] C. Löh. $\ell^{1}$-Homology and Simplicial Volume. PhD thesis, WWU Münster, 2007. http://nbn-resolving.de/urn:nbn:de:hbz:6-37549578216 Cited on page: 21 27

[18] C. Löh. Simplicial Volume. Bull. Man. Atl., pp. 7-18, 2011 Cited on page: 450

[19] C. Löh, R. Sauer. Degree theorems and Lipschitz simplicial volume for non-positively curved manifolds of finite volume. Journal of Topology, 2, pp. 193-225, 2009. Cited on page: 2

[20] B. Martelli. Complexity of PL manifolds. Algebr. Geom. Topol., 10, pp. 1107-1164, 2010. Cited on page: 5

[21] B. Martelli. Complexity of 3-manifolds. Spaces of Klenian groups. London Math. Soc. Lec. Notes Ser. 329, pp. 91-120, 2006. Cited on page:

[22] B. Martelli and C. Petronio. Complexity of geometric three-manifolds. Geometriae Dedicata, 108, pp. 15-69, 2004. Cited on page:29

[23] S. Matveev. Complexity theory of three-dimensional manifolds. Acta Appl. Math, 19, pp. 101-130, 1990. Cited on page: 5

[24] S. Matveev. Algorithmic Topology and classification of 3-manifolds. Algorithmic and computations in Mathematics, 9, Springer-Verlag, Berlin, 2003. Cited on page: 5

[25] T.S. Mrowka and P.S. Ozsváth. Low Dimensional Topology. Lecture notes from the 15th Park City Mathematics Institute (PCMI) Graduate Summer School held in Park City, UT, Summer 2006. City Mathematics Series, 15, American Mathematical Society, Providence, 2009. Cited on page: 6

[26] W. D. Neumann and A. W. Reid. Arithmetic of hyperbolic manifolds. Topology '90 (Columbus, OH, 1990), Ohio State Univ. Math. Res. Inst. Publ., 1, de Gruyter, Berlin, pp.273-310, 1992. Cited on page: 6

[27] R. Sauer. Amenable covers, volume and $L^{2}$-Betti numbers of aspherical manifolds. J. reine angew. Math., 636, pp. 47-92, 2009. Cited on page: 1

[28] M. Schmidt. $L^{2}$-Betti Numbers of $\mathcal{R}$-spaces and the Integral Foliated Simplicial Volume. PhD thesis, Westfälische Wilhelms-Universität Münster, 2005. http://nbn-resolving.de/urn:nbn:de:hbz:6-05699458563 Cited on page: 10 0 10 11. 2127 
[29] C. Strohm (= C. Löh). The Proportionality Principle of Simplicial Volume. Diplomarbeit, WWU Münster, 2004. arXiv:math.AT/0504106 Cited on page: 2

[30] M. Stroppel. Locally Compact Groups. EMS Textbooks in Mathematics. Eur. Math. Soc., Zürich Cited on page: 20

[31] W. P. Thurston. The geometry and topology of 3-manifolds. Mimeographed notes, 1979. Cited on page: 2 , 6

[32] V. S. Varadarajan. Groups of automorphisms of Borel spaces. Trans. Amer. Math. Soc., 109, p. 109-220, 1963. Cited on page: 13

[33] C. T. C. Wall. Surgery on Compact Manifolds, London Mathematical Society Monographs, no. 1, Academic Press, 1970. Cited on page: 10

FAkultät für Mathematik, Universität Regensburg, 93040 RegensburG,

E-mail address: clara.loeh@mathematik.uni-r.de

Department Mathematik, ETH Zentrum, 8092 Zürich,

E-mail address: cristina.pagliantini@math.ethz.ch 